\title{
Measuring the trilinear couplings of MSSM neutral Higgs bosons at high-energy $e^{+} e^{-}$colliders
}

\author{
P. Osland \\ Department of Physics, University of Bergen, N-5007 Bergen, Norway* \\ and Deutsches Elektronen-Synchrotron DESY, D-22603 Hamburg, Germany \\ and Theoretical Physics Division, CERN, CH-1211 Geneva 23, Switzerland \\ P. N. Pandita \\ Department of Physics, University of Bergen, N-5007 Bergen, Norway \\ and Department of Physics, North Eastern Hill University, Shillong 793 022, India*
}

(Received 12 June 1998; published 5 February 1999)

\begin{abstract}
We present a detailed analysis of multiple production of the lightest $C P$-even Higgs boson $(h)$ of the minimal supersymmetric standard model (MSSM) at high-energy $e^{+} e^{-}$colliders. We consider the production of the heavier $C P$-even Higgs boson $(H)$ via Higgs-strahlung $e^{+} e^{-} \rightarrow Z H$, in association with the $C P$-odd Higgs boson $(A)$ in $e^{+} e^{-} \rightarrow A H$, or via the fusion mechanism $e^{+} e^{-} \rightarrow \nu_{e} \bar{\nu}_{e} H$, with $H$ subsequently decaying through $H \rightarrow h h$, thereby resulting in a pair of lighter Higgs bosons $(h)$ in the final state. These processes can enable one to measure the trilinear Higgs couplings $\lambda_{H h h}$ and $\lambda_{h h h}$, which can be used to theoretically reconstruct the Higgs potential. We delineate the regions of the MSSM parameter space in which these trilinear Higgs couplings could be measured at a future $e^{+} e^{-}$collider. In our calculations, we include in detail the radiative corrections to the Higgs sector of the MSSM, especially the mixing in the squark sector.
\end{abstract}

[S0556-2821(99)04203-4]

PACS number(s): 14.80.Cp, 12.60.Jv, 13.90.+i

\section{INTRODUCTION}

The Higgs potential of the standard model (SM), which is crucial in implementing the mechanism of spontaneous symmetry breaking, contains the unknown quartic coupling of the Higgs field. As a consequence, the mass of the only Higgs boson in the SM, which is determined by this quartic coupling, is not known [1]. If a Higgs boson is discovered and its mass measured, the Higgs potential of the standard model can be uniquely determined.

On the other hand, supersymmetry is at present the only known framework in which the Higgs sector of the standard model (SM), so crucial for its internal consistency, is natural [2]. The minimal version of the supersymmetric standard model (MSSM) contains two Higgs doublets $\left(H_{1}, H_{2}\right)$ with opposite hypercharges: $Y\left(H_{1}\right)=-1, Y\left(H_{2}\right)=+1$, so as to generate masses for up- and down-type quarks (and leptons), and to cancel gauge anomalies. After spontaneous symmetry breaking induced by the neutral components of $H_{1}$ and $H_{2}$ obtaining vacuum expectation values, $\left\langle H_{1}\right\rangle=v_{1},\left\langle H_{2}\right\rangle$ $=v_{2}, \quad \tan \beta=v_{2} / v_{1}$, the MSSM contains two neutral $C P$-even ${ }^{1}(h, H)$, one neutral $C P$-odd $(A)$, and two charged $\left(H^{ \pm}\right)$Higgs bosons [1]. Although gauge invariance and supersymmetry fix the quartic couplings of the Higgs bosons in the MSSM in terms of $S U(2)_{L}$ and $U(1)_{Y}$ gauge couplings, $g$ and $g^{\prime}$, respectively, there still remain two independent parameters that describe the Higgs sector of the MSSM. These are usually chosen to be $\tan \beta$ and $m_{A}$, the mass of the

\footnotetext{
*Permanent addresses.

${ }^{1}$ When unambiguous, we shall denote the $C P$-even Higgs particles as $h$ and $H$.
}

$C P$-odd Higgs boson. All the Higgs boson masses and the Higgs couplings in the MSSM can be described (at the tree level) in terms of these two parameters.

In particular, all the trilinear self-couplings of the physical Higgs particles can be predicted theoretically (at the tree level) in terms of $m_{A}$ and $\tan \beta$. Once a light Higgs boson is discovered, the measurement of these trilinear couplings can be used to reconstruct the Higgs potential of the MSSM. This will go a long way toward establishing the Higgs mechanism as the basic mechanism of spontaneous symmetry breaking in gauge theories. Although the measurement of all the Higgs couplings in the MSSM is a difficult task, preliminary theoretical investigations by Plehn, Spira and Zerwas [3], and by Djouadi, Haber and Zerwas [4] (DHZ) of the measurement of these couplings at the LHC and at a high-energy $e^{+} e^{-}$linear collider, respectively, are encouraging.

In this paper we consider in detail the question of possible measurements of the trilinear Higgs couplings of the MSSM at a high-energy $e^{+} e^{-}$linear collider. We assume that such a facility will operate at an energy of $500 \mathrm{GeV}$ with an integrated luminosity per year of $\mathcal{L}_{\text {int }}=500 \mathrm{fb}^{-1}$ [5]. (This is a factor of 10 more than the earlier estimate.) In a later phase one may envisage an upgrade to an energy of $1.5 \mathrm{TeV}$. Since the "interesting" cross sections fall off like $1 / E^{2}$, the luminosity should increase by a corresponding factor. An earlier estimated luminosity of $500 \mathrm{fb}^{-1}$ at $1.5 \mathrm{TeV}$ may turn out to be too conservative.

The trilinear Higgs couplings that are of interest are $\lambda_{H h h}, \lambda_{h h h}$, and $\lambda_{h A A}$, involving both the $C P$-even $(h, H)$ and $C P$-odd $(A)$ Higgs bosons. ${ }^{2}$ The couplings $\lambda_{H h h}$ and

\footnotetext{
${ }^{2}$ These are not the only couplings that occur in the Higgs potential. However, these are the only ones which could possibly be measured at future colliders.
} 
$\lambda_{h h h}$ are rather small with respect to the corresponding trilinear coupling $\lambda_{h h h}^{\mathrm{SM}}$ in the SM (for a given mass of the lightest Higgs boson $m_{h}$ ), unless $m_{h}$ is close to the upper value (decoupling limit). The coupling $\lambda_{h A A}$ remains small for all parameters.

Throughout, we include one-loop radiative corrections [6] to the Higgs sector in the effective potential approximation. In particular, we take into account the parameters $A$ and $\mu$, the soft supersymmetry breaking trilinear parameter and the bilinear Higgs(ino) parameter in the superpotential, respectively, and as a consequence the left-right mixing in the squark sector, in our calculations. We thus include all the relevant parameters of the MSSM in our study, which is more detailed than the preliminary one of DHZ.

For a given value of $m_{h}$, the values of these couplings significantly depend on the soft supersymmetry-breaking trilinear parameter $A$, as well as on $\mu$, and thus on the resulting mixing in the squark sector. Since the trilinear couplings tend to be small, and depend on several parameters, their effects are somewhat difficult to estimate.

The plan of the paper is as follows. In Sec. II we review the Higgs sector of the MSSM, including the radiative corrections to the masses. The trilinear couplings are presented in Sec. III. In Sec. IV we review the possible production mechanisms for the multiple production of Higgs bosons through which the trilinear Higgs couplings can be measured at an $e^{+} e^{-}$linear collider. In Sec. V we consider the dominant source of the multiple production of the Higgs $(h)$ boson through Higgs-strahlung of $H$, and through production of $H$ in association with the $C P$-odd Higgs boson $(A)$, and the background to these processes. This source of multiple production can be used to extract the trilinear Higgs coupling $\lambda_{H h h}$.

Section VI deals with a detailed calculation of the cross section for the double Higgs-strahlung process $e^{+} e^{-}$ $\rightarrow Z h h$. This process involves the trilinear couplings $\lambda_{H h h}$ and $\lambda_{h h h}$ of the $C P$-even Higgs bosons $(h, H)$. In Sec. VII we consider the different fusion mechanisms for multiple $h$ production, especially the non-resonant process $e^{+} e^{-}$ $\rightarrow \nu_{e} \bar{\nu}_{e} h h$, for which we present a detailed calculation of the cross section in the "effective $W W$ approximation." This process also involves the two trilinear Higgs couplings, $\lambda_{H h h}$ and $\lambda_{h h h}$, and is the most useful one for extracting the coupling $\lambda_{h h h}$. In Sec. VIII we present, based on our calculations, the regions of the MSSM parameter space in which the trilinear couplings $\lambda_{H h h}$ and $\lambda_{h h h}$ could be measured; finally, in Sec. IX we present a summary of our results and conclusions.

\section{THE HIGGS SECTOR OF THE MSSM}

In this section we review the Higgs sector of the minimal supersymmetric standard model in order to set the notation and to describe the approximations we use in our calculations. As mentioned in the Introduction, we shall include the dependence on the parameters $A$ and $\mu$ through mixing in the squark sector. Where there is an overlap, our notation and approach closely follow those of Ref. [4].

At the tree level, the Higgs sector of the MSSM is de- scribed by two parameters, which can be conveniently chosen as $m_{A}$ and $\tan \beta$ [1]. There are, however, substantial radiative corrections to the $C P$-even neutral Higgs masses and couplings [6]. In the one-loop effective potential approximation, the radiatively corrected squared-mass matrix for the $C P$-even Higgs bosons can be written as [7]

$$
\begin{aligned}
\mathcal{M}^{2}= & {\left[\begin{array}{cc}
m_{A}^{2} \sin ^{2} \beta+m_{Z}^{2} \cos ^{2} \beta & -\left(m_{Z}^{2}+m_{A}^{2}\right) \sin \beta \cos \beta \\
-\left(m_{Z}^{2}+m_{A}^{2}\right) \sin \beta \cos \beta & m_{A}^{2} \cos ^{2} \beta+m_{Z}^{2} \sin ^{2} \beta
\end{array}\right] } \\
& +\frac{3 g^{2}}{16 \pi^{2} m_{W}^{2}}\left[\begin{array}{ll}
\Delta_{11} & \Delta_{12} \\
\Delta_{12} & \Delta_{22}
\end{array}\right],
\end{aligned}
$$

where the second matrix represents the radiative corrections. $^{3}$

The functions $\Delta_{i j}$ depend, besides the top- and bottomquark masses, on the Higgs bilinear parameter $\mu$ in the superpotential, the soft supersymmetry-breaking trilinear couplings $\left(A_{t}, A_{b}\right)$ and soft scalar masses $\left(m_{Q}, m_{U}, m_{D}\right)$, as well as on $\tan \beta$. We shall ignore the $b$-quark mass effects in $\Delta_{i j}$ in our calculations, which is a reasonable approximation for moderate values of $\tan \beta \leqslant 20-30$. Furthermore, we shall assume, as is often done,

$$
\begin{aligned}
& A \equiv A_{t}=A_{b}, \\
& \widetilde{m} \equiv m_{Q}=m_{U}=m_{D} .
\end{aligned}
$$

With these approximations we can write $\left(m_{t}\right.$ is the top quark mass) [7]

$$
\begin{aligned}
\Delta_{11}= & \frac{m_{t}^{4}}{\sin ^{2} \beta}\left(\frac{\mu(A+\mu \cot \beta)}{m_{\tilde{t}_{1}}^{2}-m_{\tilde{t}_{2}}^{2}}\right)^{2} g\left(m_{\tilde{t}_{1}}^{2}, m_{\tilde{t}_{2}}^{2}\right), \\
\Delta_{22}= & \frac{m_{t}^{4}}{\sin ^{2} \beta}\left(\log \frac{m_{\tilde{t}_{1}}^{2} m_{\tilde{t}_{2}}^{2}}{m_{t}^{4}}\right. \\
& \left.+\frac{2 A(A+\mu \cot \beta)}{m_{\tilde{t}_{1}}^{2}-m_{\tilde{t}_{2}}^{2}} \log \frac{m_{\tilde{t}_{1}}^{2}}{m_{\tilde{t}_{2}}^{2}}\right) \\
& +\frac{m_{t}^{4}}{\sin ^{2} \beta}\left(\frac{\mu(A+\mu \cot \beta)}{m_{\tilde{t}_{1}}^{2}-m_{\tilde{t}_{2}}^{2}}\right)^{2} g\left(m_{\tilde{t}_{1}}^{2}, m_{\tilde{t}_{2}}^{2}\right),
\end{aligned}
$$

\footnotetext{
${ }^{3}$ We note that two-loop corrections to the Higgs boson masses in the MSSM are sizable, especially for large mixing in the stop sector. For the dominant two-loop radiative corrections to the Higgs sector of the MSSM, see, e.g. [8]. In this paper we restrict ourselves to one-loop corrections only.
} 


$$
\begin{aligned}
\Delta_{12}= & \frac{m_{t}^{4}}{\sin ^{2} \beta} \frac{\mu(A+\mu \cot \beta)}{m_{\tilde{t}_{1}}^{2}-m_{\tilde{t}_{2}}^{2}} \\
& \times\left(\log \frac{m_{\tilde{t}_{1}}^{2}}{m_{\tilde{t}_{2}}^{2}}+\frac{A(A+\mu \cot \beta)}{m_{\tilde{t}_{1}}^{2}-m_{\tilde{t}_{2}}^{2}} g\left(m_{\tilde{t}_{1}}^{2}, m_{\tilde{t}_{2}}^{2}\right)\right),
\end{aligned}
$$

where $m_{\tilde{t}_{1}}^{2}$ and $m_{\tilde{t}_{2}}^{2}$ are squared stop masses given by

$$
m_{\tilde{t}_{1,2}}^{2}=m_{t}^{2}+\tilde{m}^{2} \pm m_{t}(A+\mu \cot \beta)
$$

(we have ignored the small $D$-term contributions to the stop masses) and

$$
g\left(m_{t_{1}}^{2}, m_{\tilde{t}_{2}}^{2}\right)=2-\frac{m_{\tilde{t}_{1}}^{2}+m_{\tilde{t}_{2}}^{2}}{m_{\tilde{t}_{1}}^{2}-m_{\tilde{t}_{2}}^{2}} \log \frac{m_{\tilde{t}_{1}}^{2}}{m_{\tilde{t}_{2}}^{2}} .
$$

The one-loop radiatively corrected masses $\left(m_{h}, m_{H} ; m_{h}\right.$ $<m_{H}$ ) of the $C P$-even Higgs bosons $(h, H)$ can be obtained by diagonalizing the $2 \times 2$ mass matrix in Eq. (2.1). The radiative corrections are, in general, positive, and they shift the mass of the lightest Higgs boson upwards from its treelevel value. We show in Fig. 1 the resulting mass of the lightest Higgs boson, $m_{h}$, as a function of $\mu$ and $\tan \beta$, for two values of $A$ and two values of $m_{A}$, and for $\tilde{m}=1 \mathrm{TeV}$. With a wider range of parameter values, or when the squark mass scale is taken to be smaller, the dependence on $\mu$ and $\tan \beta$ can be more dramatic [9].

The Higgs mass falls rapidly at small values of $\tan \beta$. Since the CERN $e^{+} e^{-}$collider LEP experiments are obtaining lower bounds on the mass of the lightest Higgs boson, they are beginning to rule out significant parts of the small$\tan \beta$ parameter space, depending on the model assumptions. For $\tan \beta>1$, ALEPH finds $m_{h}>62.5 \mathrm{GeV}$ at $95 \%$ C.L. $[10] .^{4}$ In our calculations, we shall therefore $\operatorname{take} \tan \beta=2$ to be a representative value. (For a recent discussion on how the lower allowed value of $\tan \beta$ depends on some of the model parameters, see Ref. [12].)

\section{TRILINEAR HIGGS COUPLINGS}

The trilinear Higgs couplings that are of interest can be written [13] as a sum of the tree-level coupling and one-loop radiative corrections:

$$
\lambda_{H h h}=\lambda_{H h h}^{0}+\Delta \lambda_{H h h},
$$

\footnotetext{
${ }^{4}$ Recently, a new study has been presented, with a lower limit of $m_{h}>72.2 \mathrm{GeV}$, irrespective of $\tan \beta$, and a limit of $\sim 88 \mathrm{GeV}$ for $1<\tan \beta \leqq 2[11]$.
}
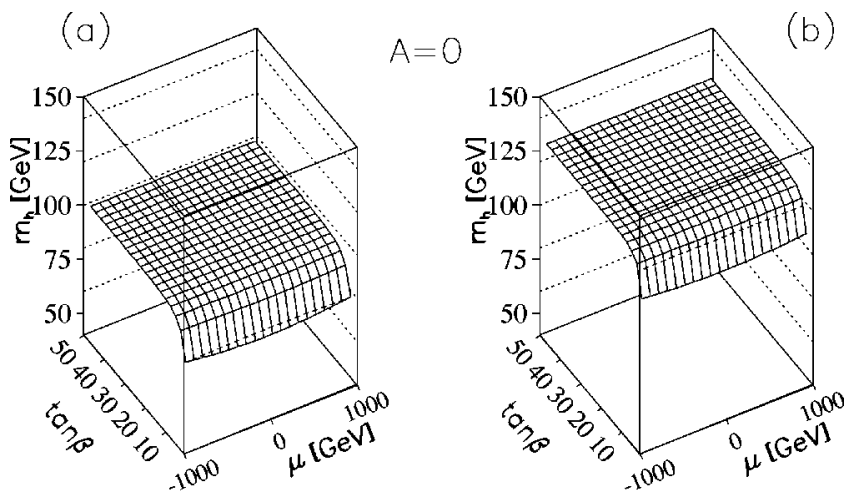

$m_{A}=100 \mathrm{GeV}$

$m_{\mathrm{A}}=400 \mathrm{GeV}$

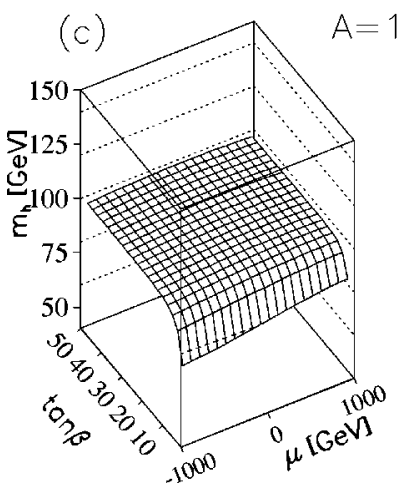

(d)

FIG. 1. Mass of the lightest Higgs boson $m_{h}$ as a function of $\mu$ and $\tan \beta$. Two values of $m_{A}$ and two values of $A$ are considered: (a) $m_{A}=100 \mathrm{GeV}, A=0$; (b) $m_{A}=400 \mathrm{GeV}, A=0 \mathrm{GeV}$; (c) $m_{A}=100 \mathrm{GeV}, A=1 \mathrm{TeV}$; (d) $m_{A}=400 \mathrm{GeV}, A=1 \mathrm{TeV}$. We have taken $\widetilde{m}=1 \mathrm{TeV}$.

$$
\begin{gathered}
\lambda_{h h h}=\lambda_{h h h}^{0}+\Delta \lambda_{h h h}, \\
\lambda_{h A A}=\lambda_{h A A}^{0}+\Delta \lambda_{h A A} .
\end{gathered}
$$

In units of $g m_{Z} /\left(2 \cos \theta_{\mathrm{W}}\right)=\left(\sqrt{2} G_{F}\right)^{1 / 2} m_{Z}^{2}$, the tree-level couplings are given by

$$
\begin{aligned}
& \lambda_{H h h}^{0}=2 \sin 2 \alpha \sin (\beta+\alpha)-\cos 2 \alpha \cos (\beta+\alpha), \\
& \lambda_{h h h}^{0}=3 \cos 2 \alpha \sin (\beta+\alpha), \\
& \lambda_{h A A}^{0}=\cos 2 \beta \sin (\beta+\alpha),
\end{aligned}
$$

with $\alpha$ the mixing angle in the $C P$-even Higgs sector, which can be calculated in terms of the parameters appearing in the $C P$-even Higgs mass matrix (2.1). The one-loop radiative corrections in Eqs. (3.1)-(3.3) are (in the above units) 


$$
\begin{aligned}
& \Delta \lambda_{H h h}=\left(\frac{3 g^{2} \cos ^{2} \theta_{W}}{16 \pi^{2}} \frac{m_{t}^{4}}{m_{W}^{4}} \frac{\sin \alpha \cos ^{2} \alpha}{\sin ^{3} \beta}\right) \\
& \times\left[3 \log \frac{m_{\tilde{t}_{1}}^{2} m_{\tilde{t}_{2}}^{2}}{m_{t}^{4}}+\left(m_{\tilde{t}_{1}}^{2}-m_{\tilde{t}_{2}}^{2}\right) C_{t}\left(E_{t}+2 F_{t}\right) \log \frac{m_{\tilde{t}_{1}}^{2}}{m_{\tilde{t}_{2}}^{2}}\right. \\
& +2\left(\frac{m_{t}^{2}}{m_{\tilde{t}_{1}}^{2}}\left[1+\left(m_{\tilde{t}_{1}}^{2}-m_{\tilde{t}_{2}}^{2}\right) C_{t} E_{t}\right]\right. \\
& \times\left[1+\left(m_{\tilde{t}_{1}}^{2}-m_{\tilde{t}_{2}}^{2}\right) C_{t} F_{t}\right]^{2} \\
& +\frac{m_{t}^{2}}{m_{\tilde{t}_{2}}^{2}}\left[1-\left(m_{\tilde{t}_{1}}^{2}-m_{t_{2}}^{2}\right) C_{t} E_{t}\right] \\
& \left.\left.\times\left[1-\left(m_{\tilde{t}_{1}}^{2}-m_{\tilde{t}_{2}}^{2}\right) C_{t} F_{t}\right]^{2}-2\right)\right], \\
& \Delta \lambda_{h h h}=\left(\frac{3 g^{2} \cos ^{2} \theta_{W}}{16 \pi^{2}} \frac{m_{t}^{4}}{m_{W}^{4}} \frac{\cos ^{3} \alpha}{\sin ^{3} \beta}\right) \\
& \times\left[3 \log \frac{m_{\tilde{t}_{1}}^{2} m_{\tilde{t}_{2}}^{2}}{m_{t}^{4}}+3\left(m_{\tilde{t}_{1}}^{2}-m_{\tilde{t}_{2}}^{2}\right) C_{t} F_{t} \log \frac{m_{\tilde{t}_{1}}^{2}}{m_{\tilde{t}_{2}}^{2}}\right. \\
& +2\left(\frac{m_{t}^{2}}{m_{\tilde{t}_{1}}^{2}}\left[1+\left(m_{\tilde{t}_{1}}^{2}-m_{\tilde{t}_{2}}^{2}\right) C_{t} F_{t}\right]^{3}\right. \\
& \left.\left.+\frac{m_{t}^{2}}{m_{\tilde{t}_{2}}^{2}}\left[1-\left(m_{\tilde{t}_{1}}^{2}-m_{\tilde{t}_{2}}^{2}\right) C_{t} F_{t}\right]^{3}-2\right)\right] \text {, } \\
& \Delta \lambda_{h A A}=\left(\frac{3 g^{2} \cos ^{2} \theta_{W}}{16 \pi^{2}} \frac{m_{t}^{4}}{m_{W}^{4}} \frac{\cos \alpha \cos ^{2} \beta}{\sin ^{3} \beta}\right) \\
& \times\left[\log \frac{m_{\tilde{t}_{1}}^{2} m_{\tilde{t}_{2}}^{2}}{m_{t}^{4}}+\left(m_{\tilde{t}_{1}}^{2}-m_{\tilde{t}_{2}}^{2}\right)\left(D_{t}^{2}+C_{t} F_{t}\right) \log \frac{m_{\tilde{t}_{1}}^{2}}{m_{\tilde{t}_{2}}^{2}}\right. \\
& \left.+\left(m_{\tilde{t}_{1}}^{2}-m_{\tilde{t}_{2}}^{2}\right)^{2} C_{t} D_{t}^{2} F_{t} g\left(m_{\tilde{t}_{1}}^{2}, m_{\tilde{t}_{2}}^{2}\right)\right],
\end{aligned}
$$

where

$$
\begin{aligned}
& C_{t}=(A+\mu \cot \beta) /\left(m_{\tilde{t}_{1}}^{2}-m_{\tilde{t}_{2}}^{2}\right), \\
& D_{t}=(A-\mu \tan \beta) /\left(m_{\tilde{t}_{1}}^{2}-m_{\tilde{t}_{2}}^{2}\right), \\
& E_{t}=(A+\mu \cot \alpha) /\left(m_{\tilde{t}_{1}}^{2}-m_{\tilde{t}_{2}}^{2}\right), \\
& F_{t}=(A-\mu \tan \alpha) /\left(m_{\tilde{t}_{1}}^{2}-m_{\tilde{t}_{2}}^{2}\right),
\end{aligned}
$$
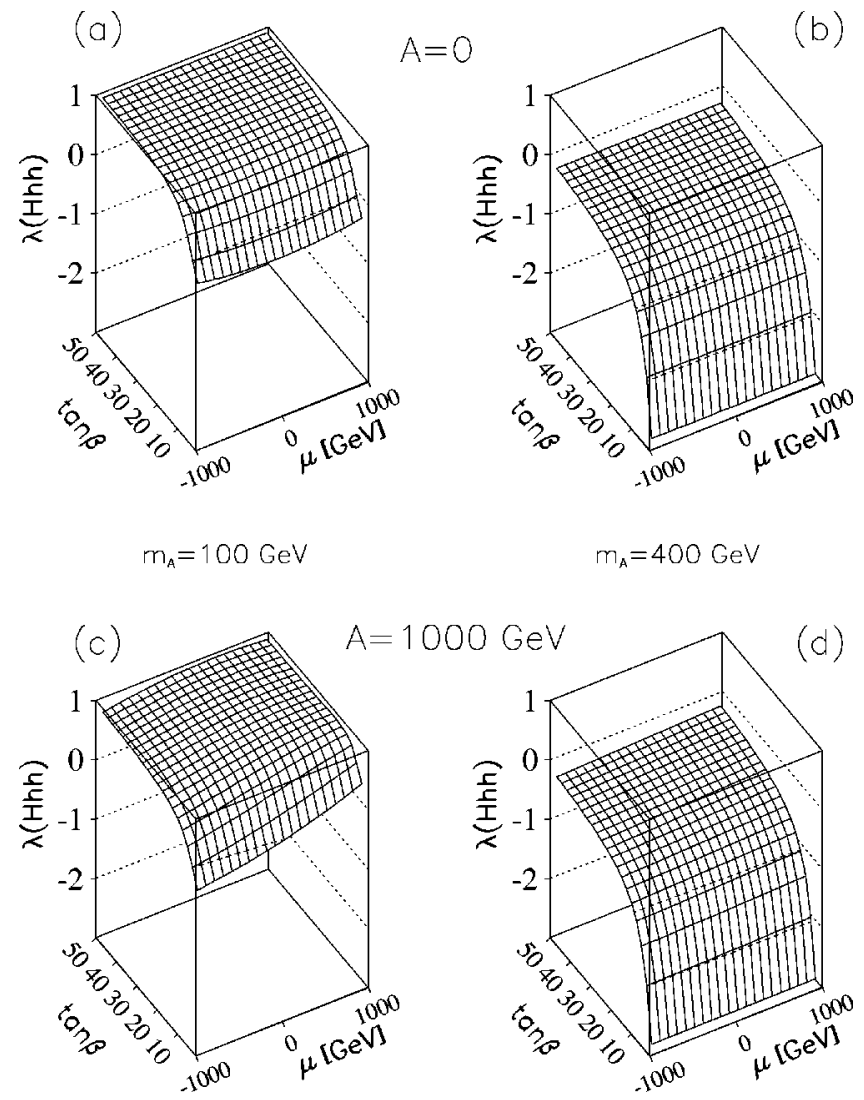

FIG. 2. Trilinear Higgs coupling $\lambda_{H h h}$ as a function of $\mu$ and $\tan \beta$. The values of the parameters are the same as in Fig. 1 .

and we have ignored the contributions from $b$-quarks and $b$-squarks, which are in general small with respect to those arising from $t$-quarks and $t$-squarks. We have also adopted the simplification described in Eq. (2.2) in writing the above results. We shall make these approximations throughout this paper.

We show in Figs. 2, 3 and 4 the couplings $\lambda_{H h h}, \lambda_{h h h}$ and $\lambda_{h A A}$ as functions of $\mu$ and $\tan \beta$, for two values of $A$ and two values of $m_{A}$, all for $\widetilde{m}=1 \mathrm{TeV}$. The explicit dependence on $A$ and $\mu$ is not dramatic, but it should be kept in mind that unless $m_{A}$ is rather small, $m_{h}$ may change considerably with $A$.

The trilinear couplings change significantly with $m_{A}$, and thus also with $m_{h}$. This is shown more explicitly in Fig. 5, where we compare $\lambda_{H h h}, \lambda_{h h h}$ and $\lambda_{h A A}$ for three different values of $\tan \beta$, and the SM quartic coupling $\lambda^{\text {SM }}$. The SM quartic coupling includes one-loop radiative corrections [14] and its normalization is such that at the tree-level, it coincides with the trilinear coupling.

At low values of $m_{h}$, the MSSM trilinear couplings are rather small. For some value of $m_{h}$ the couplings $\lambda_{H h h}$ and $\lambda_{h h h}$ start to increase in magnitude, whereas $\lambda_{h A A}$ remains small. The values of $m_{h}$ at which they start becoming significant depend crucially on $\tan \beta$. For $\tan \beta=2$ (Fig. 5(a)) this transition takes place around $m_{h} \sim 90-100 \mathrm{GeV}$, whereas for $\tan \beta=5$ and 15 , the critical values of $m_{h}$ increase to $100-110$ and $120 \mathrm{GeV}$, respectively (see Figs. 5(b) and (c)). In this region, the actual values of $\lambda_{H h h}$ and $\lambda_{h h h}$ 

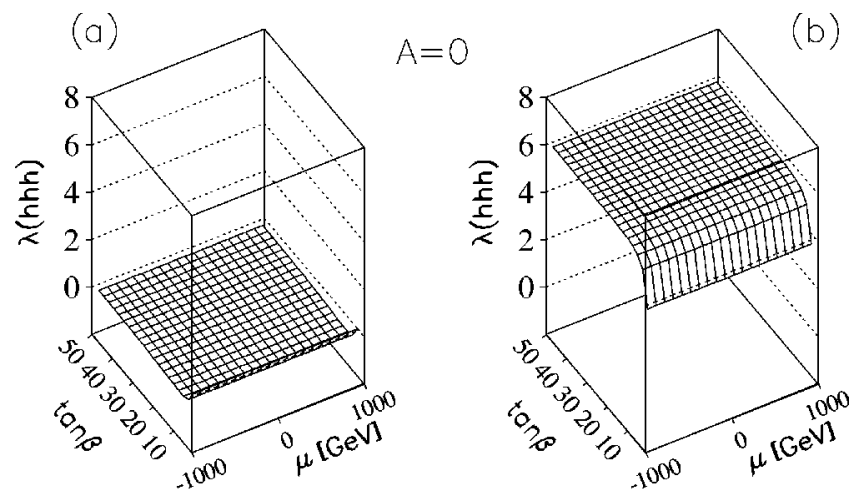

$m_{\mathrm{A}}=100 \mathrm{GeV}$

$m_{A}=400 \mathrm{GeV}$
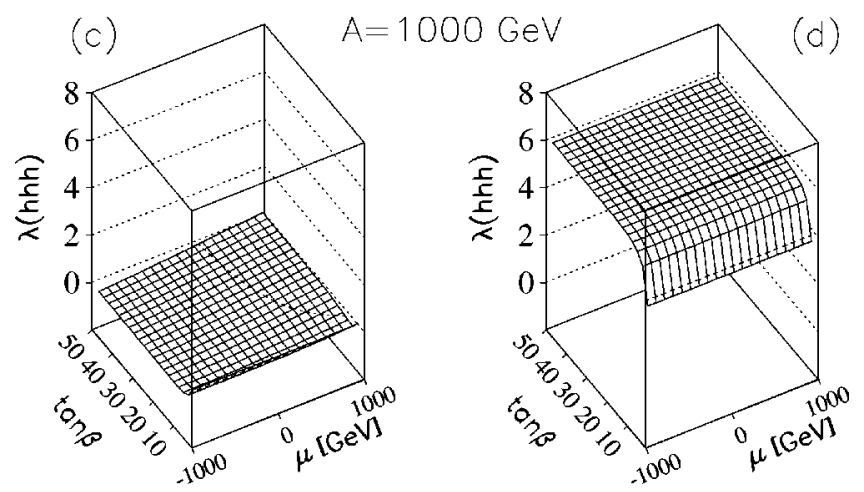

FIG. 3. Trilinear Higgs coupling $\lambda_{h h h}$ as a function of $\mu$ and $\tan \beta$. The values of the parameters are the same as in Fig. 1 .

(for a given value of $m_{h}$ ) change significantly if $A$ becomes large and positive. A non-vanishing squark-mixing parameter $A$ is thus seen to be quite important. Also, we note that for special values of the parameters, the couplings may vanish [15]. See also Fig. 1 of Ref. [3].

To sum up the behavior of the trilinear couplings, we note that $\lambda_{H h h}$ and $\lambda_{h h h}$ are small $(\leqslant 1)$ for $m_{h} \lesssim 100-120 \mathrm{GeV}$, depending on the value of $\tan \beta$. However, as $m_{h}$ approaches its maximum value, which is reached rapidly as $m_{A}$ becomes large, $m_{A} \gtrsim 200 \mathrm{GeV}$, these trilinear couplings become large $(\sim 2-4)$. Thus, as functions of $m_{A}$, the trilinear couplings $\lambda_{H h h}$ and $\lambda_{h h h}$ are large for most of the parameter space. We also note that, for large values of $\tan \beta, \lambda_{H h h}$ tends to be relatively small, whereas $\lambda_{h h h}$ becomes large, if also $m_{A}$ (or, equivalently, $m_{h}$ ) is large.

We note that for a given Higgs boson mass $m_{h}$, the tree level SM trilinear Higgs coupling is given by

$$
\lambda_{h h h}^{\mathrm{SM}}=3\left(m_{h} / m_{Z}\right)^{2}
$$

On the other hand, for large values of $m_{A}$ (the decoupling limit) the corresponding MSSM trilinear coupling, Eq. (3.5), becomes

$$
\lambda_{h h h}^{0}=3 \cos (2 \alpha) \sin (\beta+\alpha) \rightarrow 3\left(m_{h} / m_{Z}\right)^{2}
$$

i.e., it approaches the SM trilinear coupling.
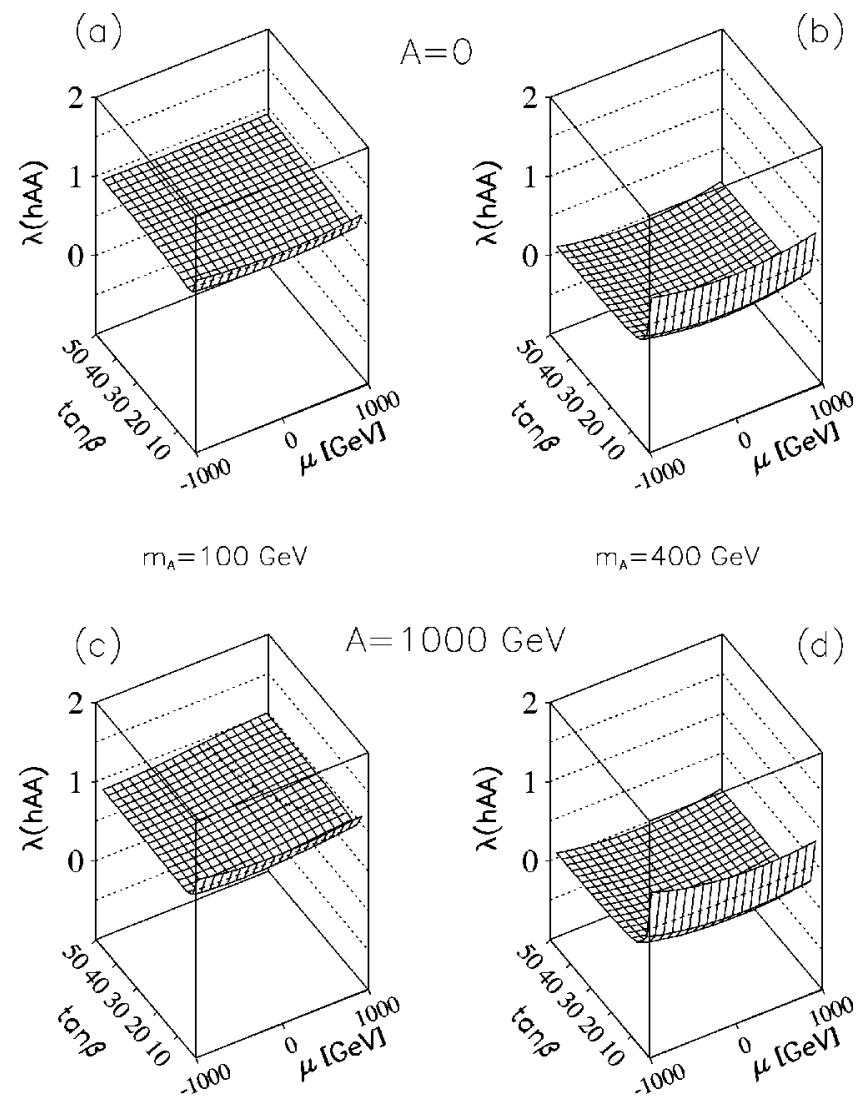

FIG. 4. Trilinear Higgs coupling $\lambda_{h A A}$ as a function of $\mu$ and $\tan \beta$. The values of the parameters are the same as in Fig. 1 .

\section{PRODUCTION MECHANISMS}

The different mechanisms for the multiple production of the MSSM Higgs bosons in $e^{+} e^{-}$collisions have been discussed by DHZ. The dominant mechanism for the production of multiple $C P$-even light Higgs bosons $(h)$ is through the production of the heavy $C P$-even Higgs boson $H$, which then decays by $H \rightarrow h h$. The heavy Higgs boson $H$ can be produced by $H$-strahlung, in association with $A$, and by the resonant $W W$ fusion mechanism. These mechanisms for multiple production of $h$

$$
\left.\begin{array}{l}
e^{+} e^{-} \rightarrow Z H, A H \\
e^{+} e^{-} \rightarrow \nu_{e} \bar{\nu}_{e} H
\end{array}\right\}, \quad H \rightarrow h h
$$

are shown in Fig. 6. We note that all the diagrams of Fig. 6 involve the trilinear coupling $\lambda_{H h h}$.

A background to Eq. (4.1) comes from the production of the pseudoscalar $A$ in association with $h$ and its subsequent decay to $h Z$

$$
e^{+} e^{-} \rightarrow h A, \quad A \rightarrow h Z
$$



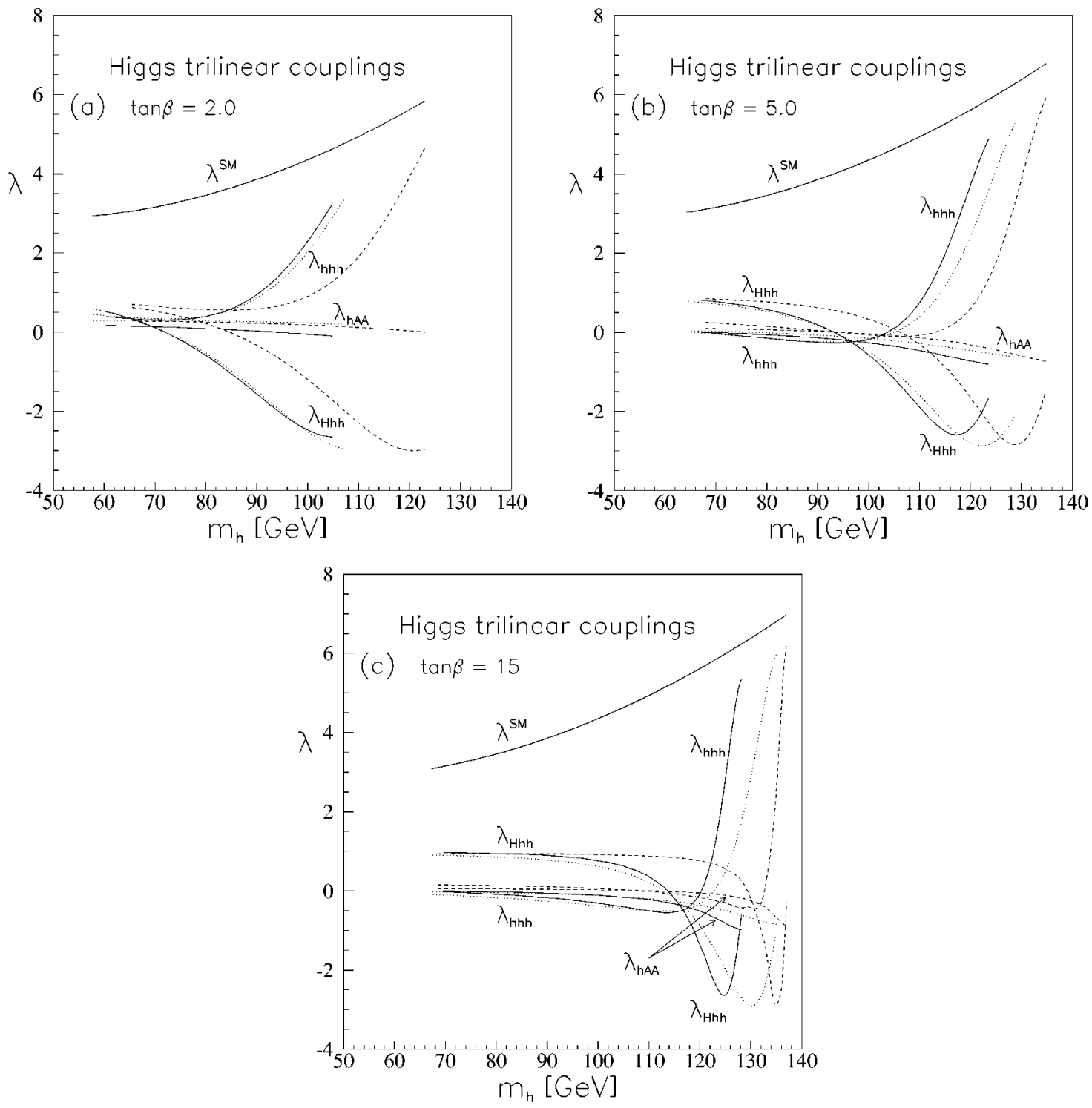

FIG. 5. Trilinear Higgs couplings $\lambda_{H h h}, \lambda_{h h h}$ and $\lambda_{h A A}$ as functions of $m_{h}$ for three values of $\tan \beta$ : (a) $\tan \beta=2.0$; (b) $\tan \beta=5.0$; (c) $\tan \beta=15$. Each coupling is shown for three cases of the mixing parameters: no mixing $(A=0, \mu=0$, solid), mixing with $A=1 \mathrm{TeV}$ and $\mu=-1 \mathrm{TeV}$ (dotted), as well as $A=1 \mathrm{TeV}$ and $\mu=1 \mathrm{TeV}$ (dashed). For comparison the SM quartic coupling $\lambda^{\mathrm{SM}}$ is also shown.

A second mechanism for $h h$ production is double Higgsstrahlung in the continuum with a $Z$ boson in the final state (see Fig. 7(a)-(d)),

$$
e^{+} e^{-} \rightarrow Z^{*} \rightarrow Z h h
$$

(a)

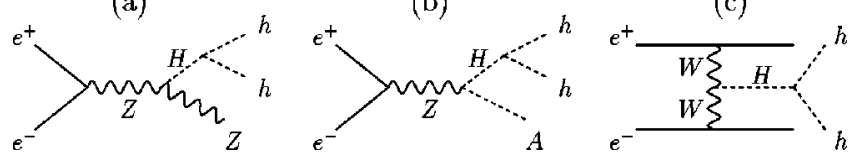

FIG. 6. Feynman diagrams for the resonant production of $h h$ final states in $e^{+} e^{-}$collisions. Diagrams (a) and (b) represent the production of $H$ in association with $Z$ and $A$, respectively, whereas diagram (c) is the $W W$ fusion mechanism for the production of $H$. The Higgs boson $H$ decays via $H \rightarrow h h$ to produce the two-Higgs final state.
We note that the Feynman diagram of Fig. 7(c) involves, apart from the coupling $\lambda_{H h h}$, the trilinear Higgs coupling $\lambda_{h h h}$ as well, whereas the other diagrams do not involve any of the trilinear Higgs couplings.

A third way of generating multiple Higgs bosons in $e^{+} e^{-}$ collisions is through associated production of $(h h)$ with the pseudoscalar $A$ in the continuum (see Fig. 8):

$$
e^{+} e^{-} \rightarrow Z^{*} \rightarrow h h A
$$

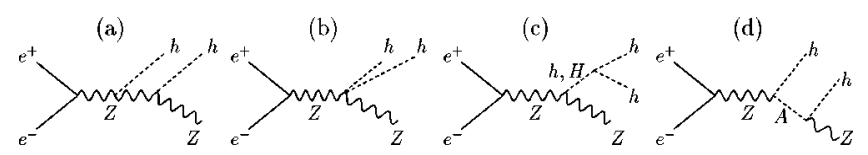

FIG. 7. Feynman diagrams for the non-resonant production of $h h$ final states in association with $Z$. The diagram (d), with $A$ produced on the mass shell, which subsequently decays via $A \rightarrow h Z$, is a background to the resonance process, Fig. 6(a). 
(a)
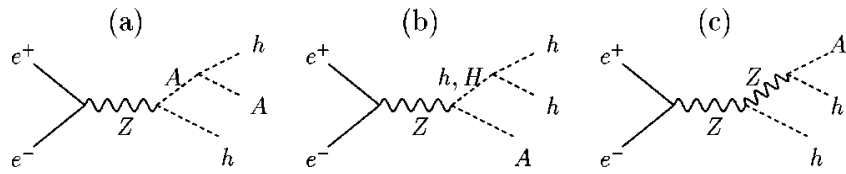

FIG. 8. Feynman diagrams for the associated production of $h h$ with the pseudoscalar $A$ in the continuum.

This process will be briefly discussed in Sec. VI. It involves, besides $\lambda_{H h h}$ and $\lambda_{h h h}$, the trilinear coupling $\lambda_{h A A}$ as well. It is, however, difficult [4] to measure this coupling $\lambda_{h A A}$ through the process (4.4).

Finally, there is a mechanism of multiple production of the lightest Higgs boson through non-resonant $W W(Z Z)$ fusion in the continuum (see Fig. 9):

$$
e^{+} e^{-} \rightarrow \bar{\nu}_{e} \nu_{e} W^{*} W^{*} \rightarrow \bar{\nu}_{e} \nu_{e} h h
$$

which will be discussed in Sec. VII.

It is important to note that all the diagrams of Fig. 6 involve the trilinear coupling $\lambda_{H h h}$ only. On the other hand, Fig. 7(c), Fig. 8(b) and Fig. 9(c) all involve both the trilinear Higgs couplings $\lambda_{H h h}$ and $\lambda_{h h h}$.

\section{HIGGS-STRAHLUNG AND ASSOCIATED PRODUCTION OF $H$}

As stated in Sec. IV, the dominant source for the production of multiple Higgs bosons $(h)$ in $e^{+} e^{-}$collisions is through the production of the heavier $C P$-even Higgs boson $H$ either via Higgs-strahlung or in association with $A$ [1], followed, if kinematically allowed, by the cascade decay $H$ $\rightarrow h h$. In terms of the $Z$-electron couplings $v_{e}=-1$ $+4 \sin ^{2} \theta_{W}, a_{e}=-1$, the cross sections for these processes can be written as $[16,17]$

$$
\begin{aligned}
\sigma\left(e^{+} e^{-} \rightarrow Z H\right)= & \frac{G_{F}^{2} m_{Z}^{4}}{96 \pi s}\left(v_{e}^{2}+a_{e}^{2}\right) \cos ^{2}(\beta-\alpha) \\
& \times \frac{\lambda_{Z}^{1 / 2}\left[\lambda_{Z}+12 m_{Z}^{2} / s\right]}{\left(1-m_{Z}^{2} / s\right)^{2}}, \\
\sigma\left(e^{+} e^{-} \rightarrow A H\right)= & \frac{G_{F}^{2} m_{Z}^{4}}{96 \pi s}\left(v_{e}^{2}+a_{e}^{2}\right) \sin ^{2}(\beta-\alpha) \\
& \times \frac{\lambda_{A}^{3 / 2}}{\left(1-m_{Z}^{2} / s\right)^{2}},
\end{aligned}
$$

where $\lambda_{j}$ refers to $\lambda\left(m_{j}^{2}, m_{H}^{2} ; s\right)$, the two-body phase-space function, and is given as

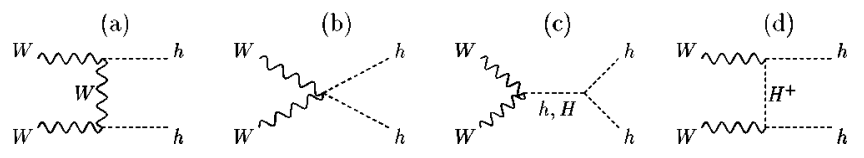

FIG. 9. Feynman diagrams for the non-resonant $W W$ fusion mechanism for the production of $h h$ states in $e^{+} e^{-}$collisions.

$$
\lambda\left(m_{a}^{2}, m_{b}^{2} ; m_{c}^{2}\right)=\left(1-\frac{m_{a}^{2}}{m_{c}^{2}}-\frac{m_{b}^{2}}{m_{c}^{2}}\right)^{2}-\frac{4 m_{a}^{2} m_{b}^{2}}{m_{c}^{4}} .
$$

In Fig. 10 we plot the cross sections (5.1) and (5.2) for the $e^{+} e^{-}$center-of-mass energies $\sqrt{s}=500 \mathrm{GeV}$ and $1.5 \mathrm{TeV}$, as functions of the Higgs mass $m_{H}$ and for $\tan \beta=2.0$. For large values of the mass $m_{A}$ of the pseudoscalar Higgs boson, all the Higgs bosons, except the lightest one $(h)$, become heavy and decouple [18] from the rest of the spectrum. In this case

$$
\cos ^{2}(\beta-\alpha) \simeq \frac{m_{Z}^{4} \sin ^{2} 4 \beta}{4 m_{A}^{4}},
$$

and the associated $A H$ production (5.2) becomes the dominant production mechanism for $H$.

At values of $\tan \beta$ that are not too large, the trilinear $H h h$ coupling $\lambda_{H h h}$ can be measured by the decay process $H$ $\rightarrow h h$, which has a width

$$
\Gamma(H \rightarrow h h)=\frac{G_{F} \lambda_{H h h}^{2}}{16 \pi \sqrt{2}} \frac{m_{Z}^{4}}{m_{H}}\left(1-\frac{4 m_{h}^{2}}{m_{H}^{2}}\right)^{1 / 2} .
$$

However, this is possible only if the decay is kinematically allowed, and the branching ratio is sizeable. In Fig. 11 we show the branching ratios (at $\tan \beta=2$ ) for the main decay modes of the heavy $C P$-even Higgs boson as a function of the $H$ mass. Apart from the $h h$ decay mode, the other important decay modes are $H \rightarrow W W^{*}, Z Z^{*}$. (We have here disregarded decays to supersymmetric particles: charginos, stops, etc. If such particles are kinematically accessible, the $H \rightarrow h h$ and $A \rightarrow Z h$ rates could be much smaller [19].) We note that the couplings of $H$ to gauge bosons can be measured through the production cross sections for $e^{+} e^{-}$ $\rightarrow \nu_{e} \bar{\nu}_{e} H$; therefore the branching ratio $B R(H \rightarrow h h)$ can be used to measure the triple Higgs coupling $\lambda_{H h h}$.

The Higgs-strahlung process [Fig. 6(a), Eq. (5.1)] gives rise to resonant two-Higgs $[h h]$ final states. This is to be contrasted with the associated production process [Fig. 6(b), Eq. (5.2)], which typically yields three Higgs $h[h h]$ final states, since the channel $A \rightarrow h Z$ is the dominant decay mode of $A$ in the mass range of interest. The decay width for $A$ $\rightarrow h Z$ can be written as [20]

$$
\begin{aligned}
\Gamma(A \rightarrow h Z)= & \frac{G_{F}}{8 \pi \sqrt{2}} \cos ^{2}(\beta-\alpha) \\
& \times \frac{m_{Z}^{4}}{m_{A}} \lambda^{1 / 2}\left(m_{Z}^{2}, m_{h}^{2} ; m_{A}^{2}\right) \lambda\left(m_{A}^{2}, m_{h}^{2} ; m_{Z}^{2}\right),
\end{aligned}
$$

where the $\lambda$ are phase-space factors given by Eq. (5.3). In Fig. 11 we show the branching ratios for the pseudoscalar $A$ for $\tan \beta=2.0$.

A background to the multiple production of lighter Higgs bosons $h$ comes from $h h$ states generated in the sequential reaction $e^{+} e^{-} \rightarrow A h \rightarrow[Z h] h$ (see Fig. 7(d)). This is a genuine background in the sense that no Higgs self-couplings are involved. But these background events are expected to be 

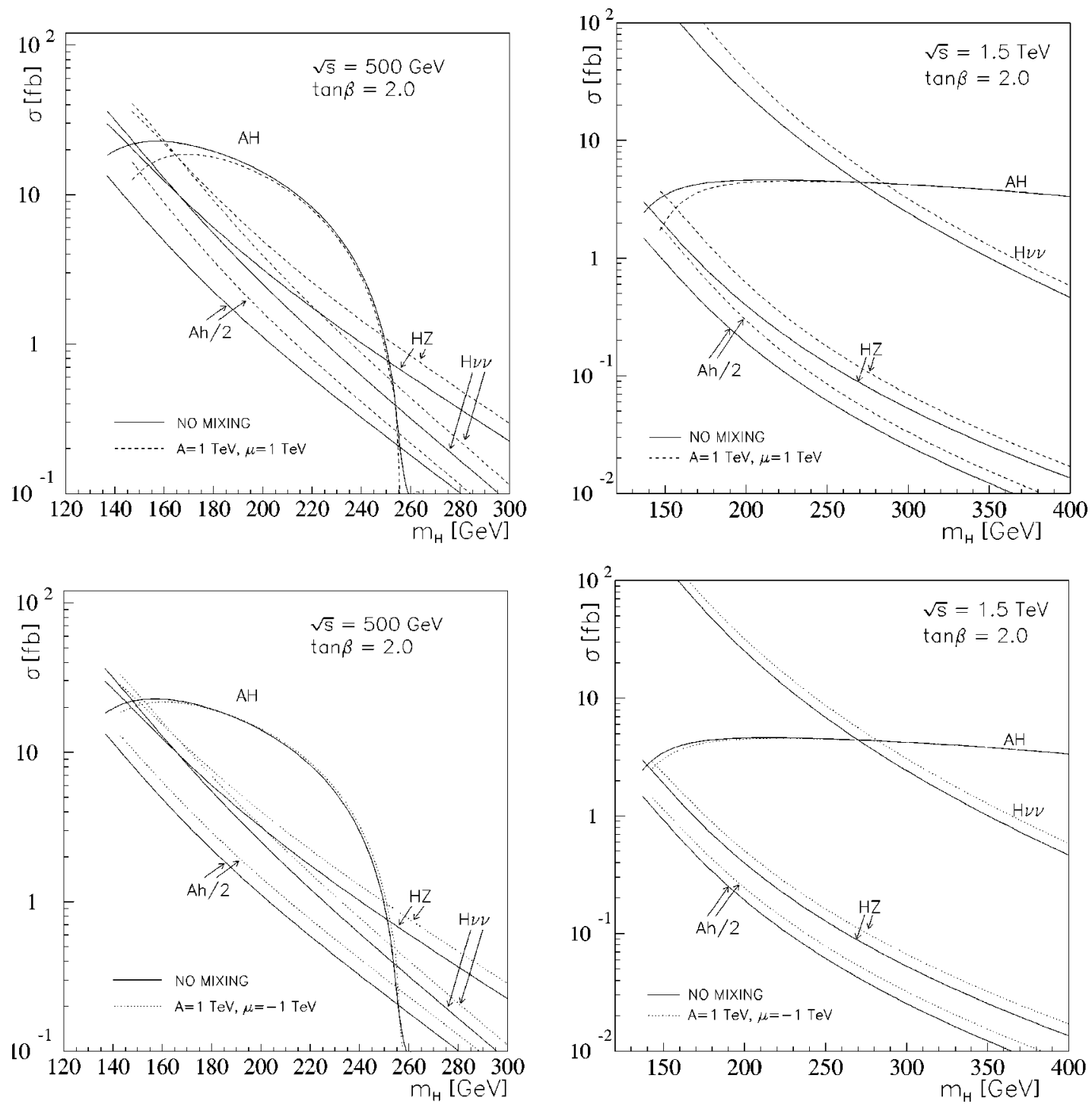

FIG. 10. Cross sections for the production of the heavy Higgs boson $H$ in $e^{+} e^{-}$collisions. Also shown is the cross section for the background process in which $A h$ is produced in the final state. We have taken $\sqrt{s}=500 \mathrm{GeV}$ and $1.5 \mathrm{TeV}$. Solid curves are for no mixing, $A=0, \mu=0$. Dashed and dotted curves refer to mixing: $A=1.0 \mathrm{TeV}, \mu=1.0 \mathrm{TeV}$ (dashed) and $A=1.0 \mathrm{TeV}, \mu=-1.0 \mathrm{TeV}$ (dotted).

topologically very different from the signal events, since the two $h$ bosons do not form a resonance, whereas the $[Z h]$ does. The cross section for the process $e^{+} e^{-} \rightarrow A h$ can be written as [17]

$$
\begin{aligned}
\sigma\left(e^{+} e^{-} \rightarrow A h\right)= & \frac{G_{F}^{2} m_{Z}^{4}}{96 \pi s}\left(v_{e}^{2}+a_{e}^{2}\right) \cos ^{2}(\beta-\alpha) \\
& \times \frac{\lambda^{3 / 2}\left(m_{h}^{2}, m_{A}^{2} ; s\right)}{\left(1-m_{Z}^{2} / s\right)^{2}},
\end{aligned}
$$

and is shown in Fig. 10 together with the signal cross sections (5.1) and (5.2). As a consequence of the decoupling theorem [18], the cross section becomes small for large values of $m_{H}$.

For increasing values of $\tan \beta$, the $H h h$ coupling gradually gets weaker (see Figs. 2 and 5), and hence the prospects for measuring $\lambda_{H h h}$ diminish. This is indicated by Fig. 12, where we show the $H$ and $A$ branching ratios for $\tan \beta=5$.

There is in fact a sizeable region in the $m_{A}-\tan \beta$ plane where the decay $H \rightarrow h h$ is kinematically forbidden. This is indicated in Fig. 13. In this figure we also display the regions where the $H \rightarrow h h$ branching ratio is in the range $0.1-0.9$. Clearly, in the forbidden region, the $\lambda_{H h h}$ cannot be determined from resonant production.

\section{DOUBLE HIGGS-STRAHLUNG AND TRIPLE $h$ PRODUCTION}

For small and moderate values of $\tan \beta$, the study of decays of the heavy $C P$-even Higgs boson $H$ provides a means of determining the triple-Higgs coupling $\lambda_{H h h}$. In order to extract the coupling $\lambda_{h h h}$, other processes involving twoHiggs $(h)$ final states must be considered. The $Z h h$ final states, which can be produced in the double Higgs-strahlung 

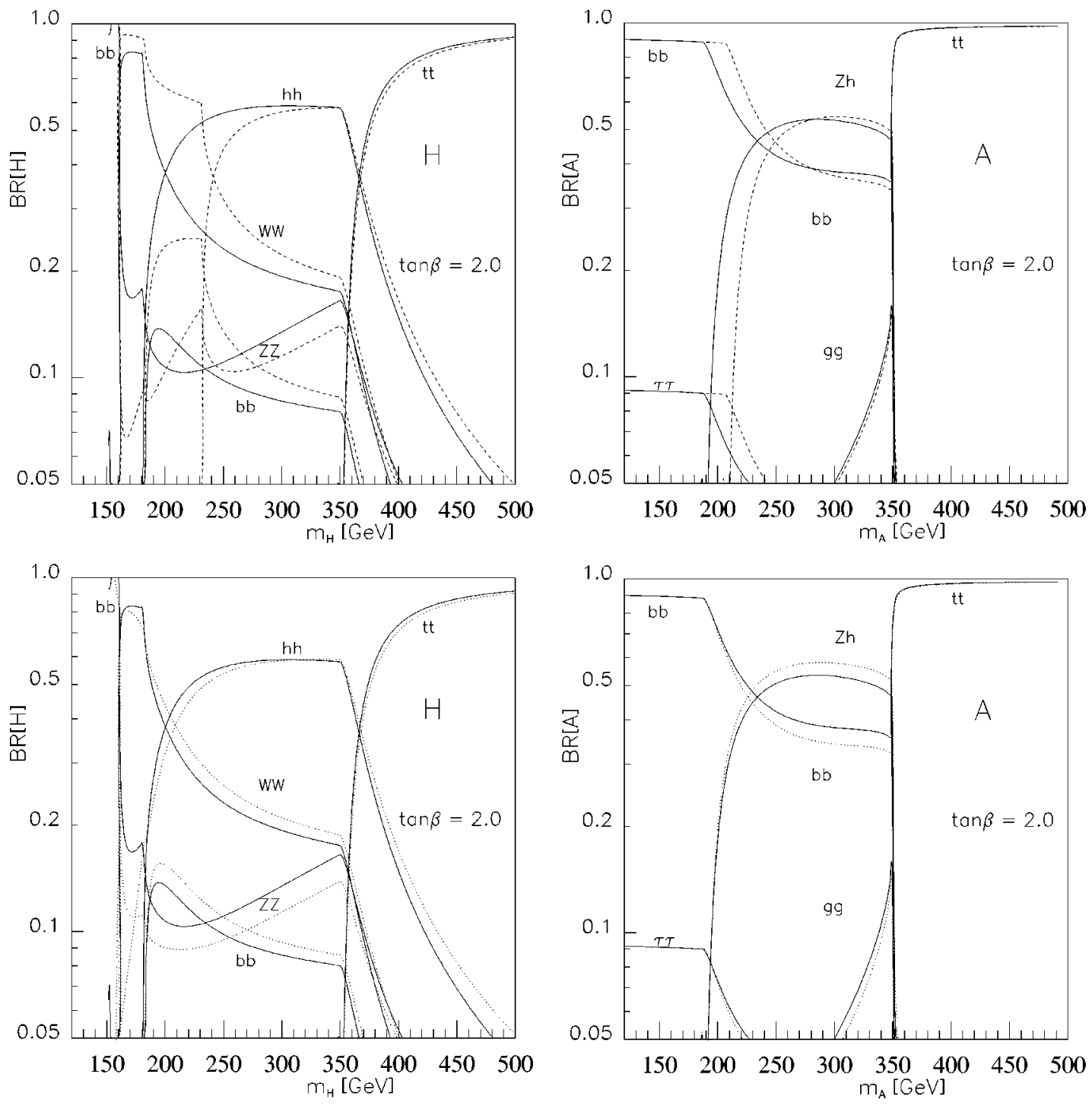

FIG. 11. Branching ratios for the decay modes of the $C P$-even heavy Higgs boson $H$, and the $C P$-odd Higgs boson $A$ for tan $\beta=2.0$. Solid curves are for no mixing, $A=0, \mu=0$. Dashed and dotted curves refer to mixing: $A=1.0 \mathrm{TeV}, \mu=1.0 \mathrm{TeV}$ (dashed) and $A$ $=1.0 \mathrm{TeV}, \mu=-1.0 \mathrm{TeV}$ (dotted).

$e^{+} e^{-} \rightarrow$ Zhh of Fig. 7, could provide one possible opportunity, since it involves the coupling $\lambda_{h h h}$ through the mechanism of Fig. 7(c). In this section we shall study these nonresonant processes in detail.

\section{A. The double Higgs-strahlung $e^{+} e^{-} \rightarrow Z h h$}

The doubly differential cross section for the process $e^{+} e^{-} \rightarrow Z h h$ shown in Fig. 7 can be written as [4]

$$
\frac{d \sigma\left(e^{+} e^{-} \rightarrow Z h h\right)}{d x_{1} d x_{2}}=\frac{G_{F}^{3} m_{Z}^{6}}{384 \sqrt{2} \pi^{3} s}\left(v_{e}^{2}+a_{e}^{2}\right) \frac{\mathcal{A}}{\left(1-\mu_{Z}\right)^{2}},
$$

where the couplings $v_{e}$ and $a_{e}$ have been defined at the beginning of Sec. V. Because of some misprints in the formulas given in [4] for the coefficient $\mathcal{A}$, we have recalculated it. Following [4], we introduce $x_{1,2}=2 E_{1,2} / \sqrt{s}$ for the scaled energies of the Higgs particles, $x_{3}=2-x_{1}-x_{2}$ for the scaled energy of the $Z$ boson, and $y_{k}=1-x_{k}$. Also, we denote by $\mu_{i}=m_{i}^{2} / s$ the scaled squared masses of various particles:

$$
\mu_{h}=m_{h}^{2} / s, \quad \mu_{H}=m_{H}^{2} / s, \quad \mu_{W}=m_{W}^{2} / s .
$$

We can express our result in a compact form as follows:

$$
\begin{aligned}
\mathcal{A}= & \mu_{Z}\left\{\frac{1}{2}|a|^{2} f_{a}+\left|b\left(y_{1}\right)\right|^{2} f_{b}+2 \operatorname{Re}\left[a b^{*}\left(y_{1}\right)\right] g_{a b}\right. \\
& \left.+\operatorname{Re}\left[b\left(y_{1}\right) b^{*}\left(y_{2}\right)\right] g_{b b}\right\}+\left\{x_{1} \leftrightarrow x_{2}\right\} .
\end{aligned}
$$

Here,

$$
\begin{aligned}
a= & \frac{1}{2}\left[\frac{\sin (\beta-\alpha) \lambda_{h h h}}{y_{3}+\mu_{Z}-\widetilde{\mu}_{h}}+\frac{\cos (\beta-\alpha) \lambda_{H h h}}{y_{3}+\mu_{Z}-\widetilde{\mu}_{H}}\right] \\
& +\left[\frac{\sin ^{2}(\beta-\alpha)}{y_{1}+\mu_{h}-\tilde{\mu}_{Z}}+\frac{\sin ^{2}(\beta-\alpha)}{y_{2}+\mu_{h}-\widetilde{\mu}_{Z}}\right]+\frac{1}{2 \mu_{Z}}
\end{aligned}
$$



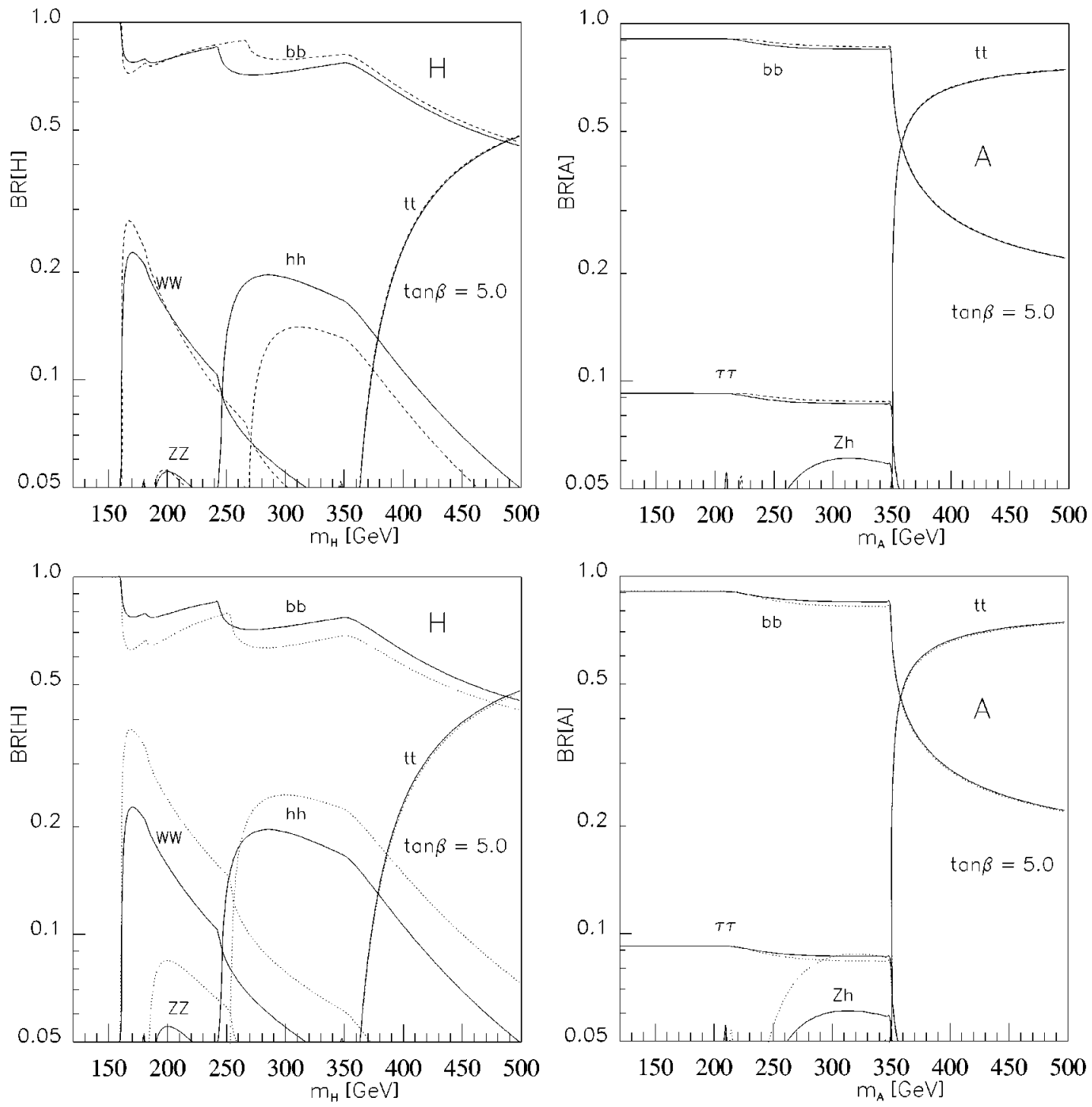

FIG. 12. As in Fig. 11, but with $\tan \beta=5.0$. Solid curves are for no mixing, $A=0, \mu=0$. Dashed and dotted curves refer to mixing: $A=1.0 \mathrm{TeV}, \mu=1.0 \mathrm{TeV}$ (dashed) and $A=1.0 \mathrm{TeV}, \mu=-1.0 \mathrm{TeV}$ (dotted).

represents a contribution from diagram $7 \mathrm{a}$, where the lepton tensor couples directly to the final-state $Z$ polarization tensor, as well as the contributions of diagrams $7 \mathrm{~b}$ and $7 \mathrm{c}$. Similarly,

$$
b(y)=\frac{1}{2 \mu_{Z}}\left(\frac{\sin ^{2}(\beta-\alpha)}{y+\mu_{h}-\tilde{\mu}_{Z}}+\frac{\cos ^{2}(\beta-\alpha)}{y+\mu_{h}-\tilde{\mu}_{A}}\right)
$$

represents the part of diagram $7 \mathrm{a}$ where the lepton tensor couples to the final-state $Z$ polarization tensor indirectly via the Higgs momenta $q_{1}$ and $q_{2}$, as well as diagram $7 \mathrm{~d}$. The tildes on $\mu_{i}$ keep track of the widths, e.g. $\tilde{\mu}_{Z}=\left(m_{Z}^{2}\right.$ $\left.+i m_{Z} \Gamma_{Z}\right) / s$.

The Higgs self-couplings $\lambda_{H h h}$ and $\lambda_{h h h}$ occur only in the function $a$, Eq. (6.4). The coefficients $f$ and $g$, which do not involve any Higgs couplings, can be expressed rather compactly as

$$
\begin{aligned}
f_{a}= & x_{3}^{2}+8 \mu_{Z}, \\
f_{b}= & \left(x_{1}^{2}-4 \mu_{h}\right)\left[\left(y_{1}-\mu_{Z}\right)^{2}-4 \mu_{Z} \mu_{h}\right] \\
g_{a b}= & \mu_{Z}\left[2\left(\mu_{Z}-4 \mu_{h}\right)+x_{1}^{2}+x_{2}\left(x_{2}+x_{3}\right)\right] \\
& -y_{1}\left(2 y_{2}-x_{1} x_{3}\right) \\
g_{b b}= & \mu_{Z}^{2}\left(4 \mu_{h}+6-x_{1} x_{2}\right)+2 \mu_{Z}\left(\mu_{Z}^{2}+y_{3}-4 \mu_{h}\right) \\
& +\left(y_{3}-x_{1} x_{2}-x_{3} \mu_{Z}-4 \mu_{h} \mu_{Z}\right) \\
& \times\left(2 y_{3}-x_{1} x_{2}-4 \mu_{h}+4 \mu_{Z}\right) .
\end{aligned}
$$

These coefficients (we use a mixed notation, which involves both $x$ and $y$ ) correspond to those of [4] as follows: $\left(f_{a}, f_{b}, g_{a b}, g_{b b}\right)=\left(f_{0}, f_{1}, f_{3}, f_{2}\right)$. With this identification, we agree with the result given in the Erratum to [4]. 

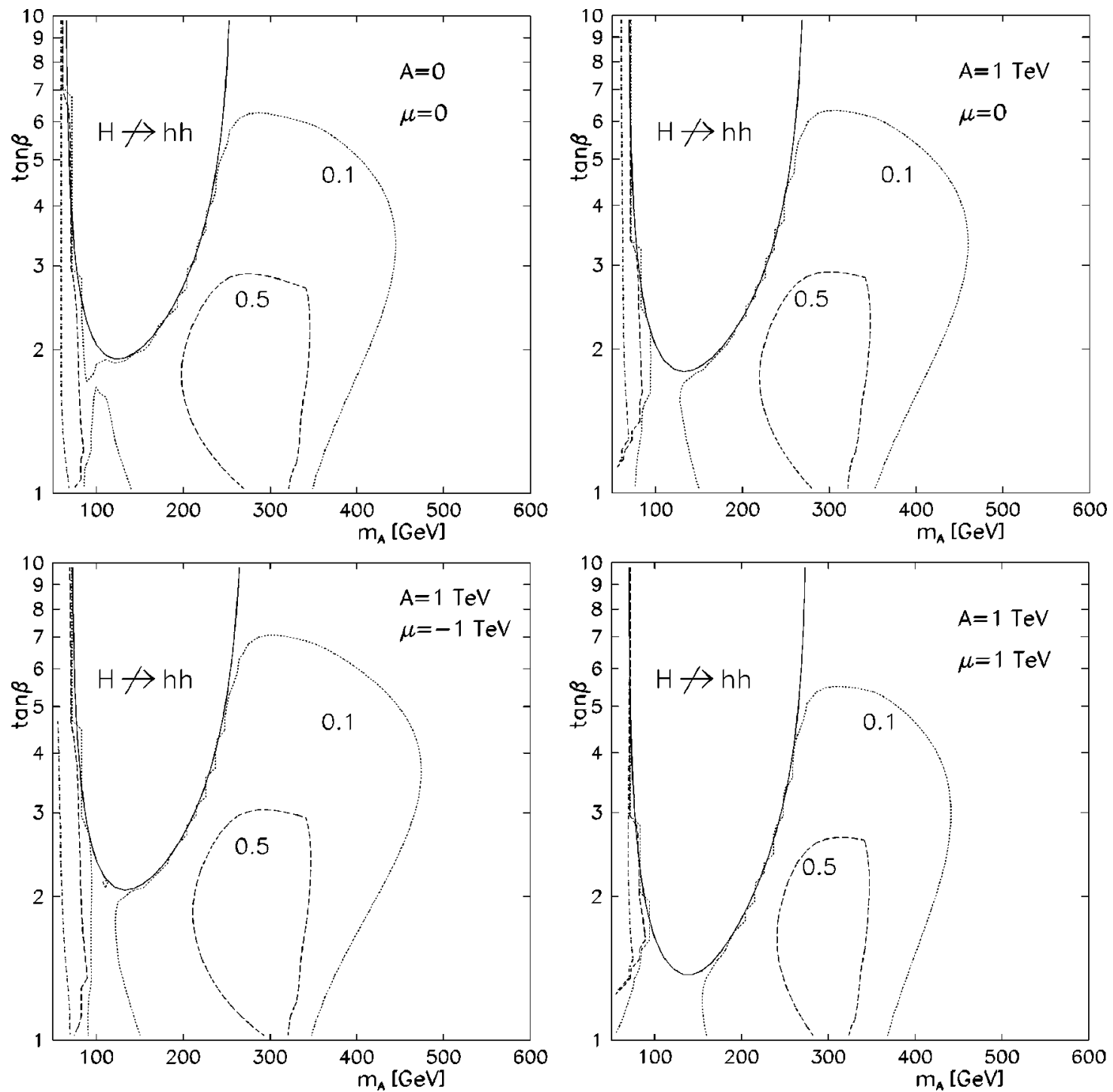

FIG. 13. The region in the $m_{A}-\tan \beta$ plane where the decay $H \rightarrow h h$ is kinematically forbidden is indicated by a solid line contour. Also given are contours at which the branching ratio equals 0.1 (dotted), 0.5 (dashed) and 0.9 (dash-dotted, at the far left). Four cases of mixing parameters $A$ and $\mu$ are considered, as indicated.

In the limit of large $m_{A}, \sin (\beta-\alpha) \rightarrow 1$, the cross section reduces to the standard model cross section with

$$
\begin{aligned}
a= & \frac{1}{2} \frac{\lambda_{h h h}}{y_{3}+\mu_{Z}-\tilde{\mu}_{h}} \\
& +\left[\frac{1}{y_{1}+\mu_{h}-\tilde{\mu}_{Z}}+\frac{1}{y_{2}+\mu_{h}-\tilde{\mu}_{Z}}\right]+\frac{1}{2 \mu_{Z}} \\
b(y)= & \frac{1}{2 \mu_{Z}} \frac{1}{y+\mu_{h}-\tilde{\mu}_{Z}},
\end{aligned}
$$

where $\lambda_{h h h} \rightarrow \lambda_{h h h}^{\mathrm{SM}}$, as discussed in Sec. III.

We show in Fig. 14(a) the Zhh cross section, as given by Eqs. (5.1), (5.5) and (6.1), in the limit of no squark mixing, and with $\widetilde{m}=1 \mathrm{TeV}$. The structure around $m_{h}=70 \mathrm{GeV}$ is due to the vanishing and near-vanishing of the trilinear coupling. In Figs. 14(b)-(d) we have introduced squark mixing: $A=1 \mathrm{TeV}, \mu=0, \pm 1 \mathrm{TeV}$. (For the decoupling-limit cross section, which is also shown, we use the MSSM coupling, instead of the SM coupling, for the reason given in Sec. III.)

In the case of no mixing, there is a broad minimum from $m_{h} \simeq 78$ to $90 \mathrm{GeV}$, followed by an enhancement around $m_{h} \sim 90-100 \mathrm{GeV}$. This structure is due to the vanishing of the branching ratio for $H \rightarrow h h$, which is kinematically forbidden in the region $m_{h} \simeq 78-90 \mathrm{GeV}$, see Fig. 13 (this coincides with the opening up of the channel $H \rightarrow W W$ ), followed by an increase of the trilinear couplings. This particular structure depends considerably on the exact mass values $m_{H}$ and $m_{h}$. Thus, it depends on details of the radiative corrections and the mixing parameters $A$ and $\mu$.

The $A \rightarrow h Z$ channel contributes of the order of $20 \%$ in the region of the maximum at $m_{h}=90-100 \mathrm{GeV}$. 

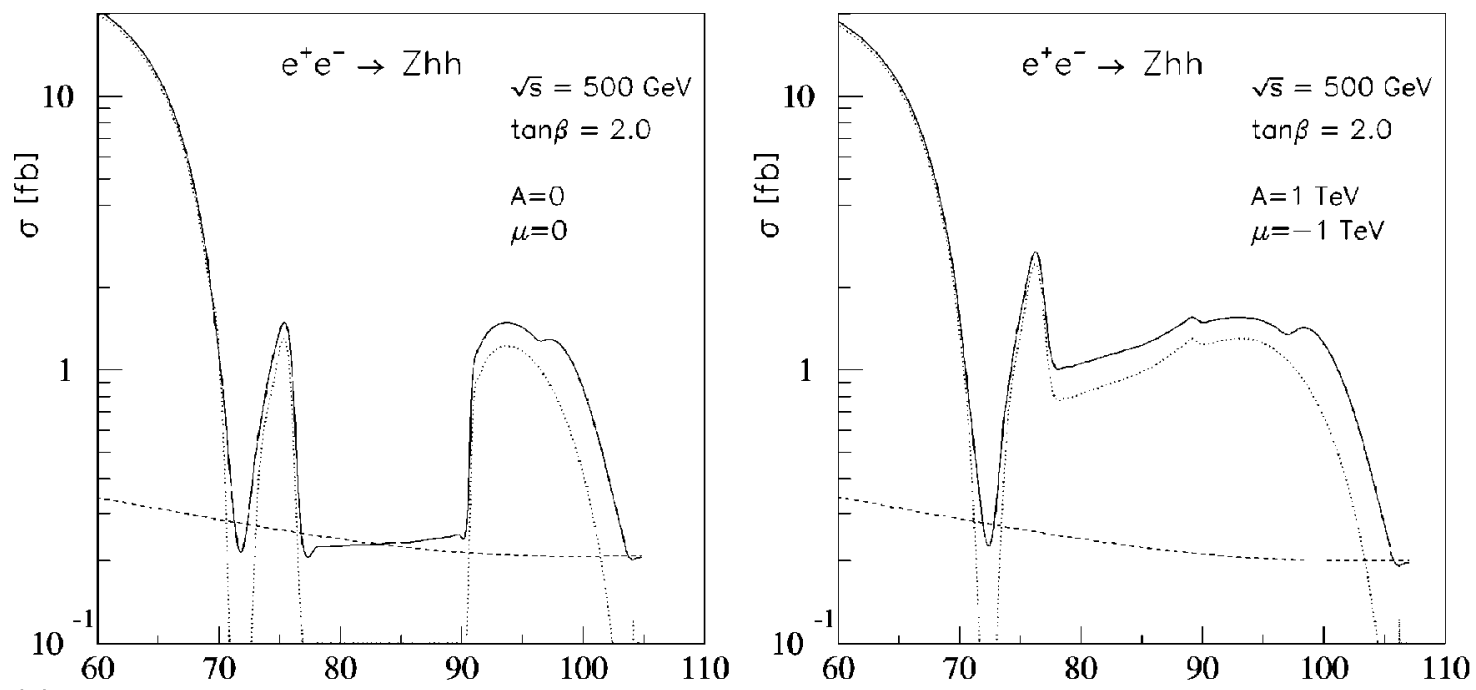

(a) $m_{h}[G e V]$

(c)
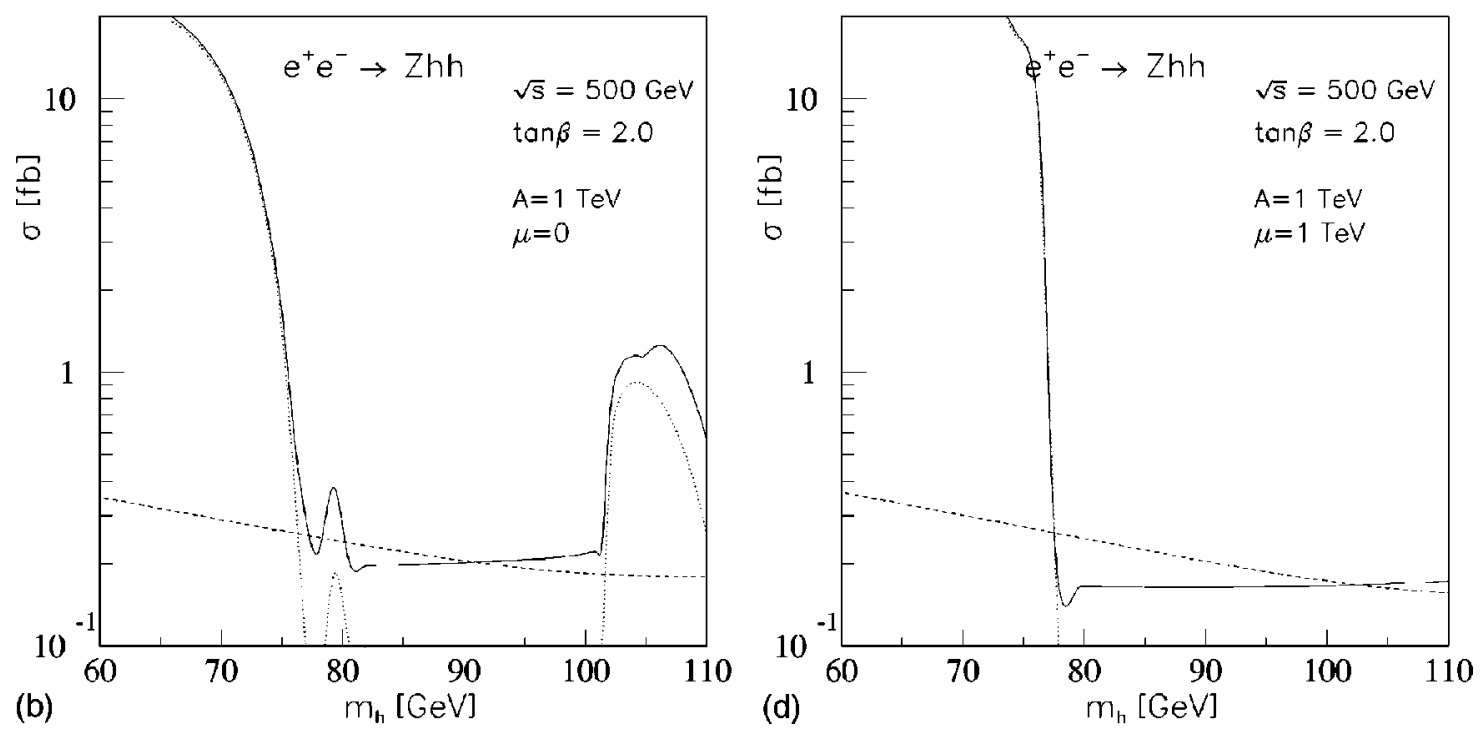

FIG. 14. Cross section $\sigma\left(e^{+} e^{-} \rightarrow Z h h\right)$ as a function of $m_{h}$, for four cases: (a) no mixing, (b) - (d) $A=1 \mathrm{TeV}, \mu=0,-1$ and $1 \mathrm{TeV}$, as indicated. The dotted curve is the resonant production. The dashed curve gives the decoupling limit.

\section{B. Triple- $h$ production}

The resonant and non-resonant production of $A h h$ (Fig. 8) can lead to three- $h$ final states in the region of $m_{A}$, where $A$ has a significant branching ratio for decaying to $Z h$, i.e. for $m_{A}$ below the $t \bar{t}$ threshold, and relatively low values of $\tan \beta$ (cf. Figs. 11 and 12).

In principle, this channel allows for a study of the coupling $\lambda_{h A A}$ (cf. Fig. 8(a)). However, the prospects for measuring this coupling, which is rather small (see Fig. 4), was studied in Ref. [4] and found not to be very encouraging.

\section{FUSION MECHANISM FOR MULTIPLE- $h$ PRODUCTION}

As mentioned in Sec. IV, a double Higgs $(h h)$ final state in $e^{+} e^{-}$collisions can also result from the $W W$ fusion mechanism, which can either be a resonant process as in Eq. (4.1), or a non-resonant one like Eq. (4.5). Since the neutralcurrent couplings are smaller than the charged-current ones, the cross section for the $Z Z$ fusion mechanism in Eqs. (4.1) and (4.5) is an order of magnitude smaller than the $W W$ fusion mechanism. We shall thus, in the following, ignore the $Z Z$ fusion mechanism, and concentrate instead on the $W W$ mechanism.

\section{A. Resonant $W W$ fusion}

The $W W$ fusion mechanism provides another large cross section for the multiple production of $h$ bosons. The cross section for $e^{+} e^{-} \rightarrow H \bar{\nu}_{e} \nu_{e}$ can be written as [21]

$$
\begin{aligned}
& \sigma\left(e^{+} e^{-} \rightarrow H \bar{\nu}_{e} \nu_{e}\right)=\frac{G_{F}^{3} m_{W}^{4}}{64 \sqrt{2} \pi^{3}} \\
& \quad \times\left[\int_{\mu_{H}}^{1} d x \int_{x}^{1} \frac{d y}{\left[1+(y-x) / \mu_{W}\right]^{2}} \mathcal{F}(x, y)\right] \\
& \quad \times \cos ^{2}(\beta-\alpha),
\end{aligned}
$$


where

$$
\begin{aligned}
\mathcal{F}(x, y)= & 16[F(x, y)+G(x, y)], \\
F(x, y)= & {\left[\frac{2 x}{y^{3}}-\frac{1+2 x}{y^{2}}+\frac{2+x}{2 y}-\frac{1}{2}\right] } \\
& \times\left[\frac{z}{1+z}-\log (1+z)\right] \\
& +\frac{x}{y^{3}} \frac{z^{2}(1-y)}{(1+z)}, \\
G(x, y)= & {\left[-\frac{x}{y^{2}}+\frac{2+x}{2 y}-\frac{1}{2}\right] } \\
& \times\left[\frac{z}{1+z}-\log (1+z)\right],
\end{aligned}
$$

with $\mu_{i}$ defined by Eq. (6.2) and

$$
z=\frac{y\left(x-\mu_{H}\right)}{\mu_{W} x} \text {. }
$$

For $\sqrt{s}, m_{H} \gg m_{W}$, and in the effective longitudinal $W$ approximation, the cross section (7.1) for $e^{+} e^{-} \rightarrow H \bar{\nu}_{e} \nu_{e}$ can be written in the following simple form [22]:

$$
\begin{aligned}
\sigma\left(e^{+} e^{-} \rightarrow H \bar{\nu}_{e} \nu_{e}\right)= & \frac{G_{F}^{3} m_{W}^{4}}{4 \sqrt{2} \pi^{3}}\left[\left(1+\frac{m_{H}^{2}}{s}\right) \log \frac{s}{m_{H}^{2}}\right. \\
& \left.-2\left(1-\frac{m_{H}^{2}}{s}\right)\right] \cos ^{2}(\beta-\alpha) .
\end{aligned}
$$

However, in this approximation the cross section may be overestimated by a factor of 2 for small values of masses and/or small center-of-mass energies. For example, at $\sqrt{s}$ $=500 \mathrm{GeV}$ the equivalent $W$ approximation gives a result that is twice as large as the exact cross section. Therefore, we use the exact cross section (7.1) in our calculations.

The cross section (7.1) is plotted in Fig. 10 for center-ofmass energies, $\sqrt{s}=500 \mathrm{GeV}$ and $1.5 \mathrm{TeV}$, and for $\tan \beta$ $=2.0$, as a function of $m_{H}$. The resonant fusion mechanism, which leads to $[h h]+[$ missing energy $]$ final states is competitive with the process $e^{+} e^{-} \rightarrow H Z \rightarrow[h h]+[$ missing energy], particularly at high energies. Since the dominant decay of $h$ will be into $b \bar{b}$ pairs, the $H$-strahlung and the fusion mechanism will give rise to final states that will predominantly include four $b$-quarks. On the other hand, the process $e^{+} e^{-} \rightarrow A H$ will give rise to six $b$-quarks in the final state, since the $A H$ final state typically yields three-Higgs $h[h h]$ final states.

\section{B. Non-resonant fusion $W W \rightarrow h h$}

Besides the resonant $W W$ fusion mechanism for the multiple production of $h$ bosons, there is also a non-resonant $W W$ fusion mechanism:

$$
e^{+} e^{-} \rightarrow \nu_{e} \bar{\nu}_{e} h h
$$

through which the same final state of two $h$ bosons can be produced. The cross section for this process, which arises through $W W$ exchange as indicated in Fig. 9, can be written in the "effective $W W$ approximation" as

$$
\sigma\left(e^{+} e^{-} \rightarrow \nu_{e} \bar{\nu}_{e} h h\right)=\int_{\tau}^{1} \mathrm{~d} x \frac{\mathrm{d} L}{\mathrm{~d} x} \hat{\sigma}_{W W}(x),
$$

where $\tau=4 m_{h}^{2} / s$. In the above, the cross section is written as a $W W$ cross section, at invariant energy squared $\hat{s}=x s$, folded with the $W W$ "luminosity", [22]:

$$
\frac{\mathrm{d} L(x)}{\mathrm{d} x}=\frac{G_{\mathrm{F}}^{2} m_{W}^{4}}{2}\left(\frac{v^{2}+a^{2}}{4 \pi^{2}}\right)^{2} \frac{1}{x}\left\{(1+x) \log \frac{1}{x}-2(1-x)\right\},
$$

where $v^{2}+a^{2}=2$.

The $W W$ cross section receives contributions from several amplitudes, according to the diagrams ${ }^{6}$ (a)-(d) in Fig. 9. We have evaluated ${ }^{7}$ these contributions and express the result in a form analogous to that of Ref. $[4]^{8}$ :

$$
\begin{aligned}
\hat{\sigma}_{W W}(x)= & \frac{G_{\mathrm{F}}^{2} \hat{s}}{64 \pi} \beta_{h}\left\{4 \left[\frac{\hat{\mu}_{Z} \sin (\beta-\alpha)}{1-\hat{\mu}_{h}} \lambda_{h h h}\right.\right. \\
& \left.+\frac{\hat{\mu}_{Z} \cos (\beta-\alpha)}{1-\hat{\mu}_{H}} \lambda_{H h h}+1\right]^{2} g_{0} \\
& +\frac{2}{\beta_{h}}\left[\frac{\hat{\mu}_{Z} \sin (\beta-\alpha)}{1-\hat{\mu}_{h}} \lambda_{h h h}\right. \\
& \left.+\frac{\hat{\mu}_{Z} \cos (\beta-\alpha)}{1-\hat{\mu}_{H}} \lambda_{H h h}+1\right] \\
& \times\left[\sin ^{2}(\beta-\alpha) g_{1}+\cos ^{2}(\beta-\alpha) g_{2}\right] \\
& +\frac{1}{\beta_{h}^{2}}\left\{\sin ^{4}(\beta-\alpha) g_{3}+\cos ^{4}(\beta-\alpha) g_{4}\right. \\
& \left.\left.+\sin ^{2}[2(\beta-\alpha)] g_{5}\right\}\right\}
\end{aligned}
$$

where we have introduced "reduced squared masses",

\footnotetext{
${ }^{5}$ There could be sizable deviations of the effective $W W$ approximation from the exact result.

${ }^{6}$ For each of the diagrams (a) and (d) there are two contributions, corresponding to the interchange of the two Higgs particles.

${ }^{7}$ There are some misprints in Ref. [4], so we present here results of an independent calculation.

${ }^{8}$ In Ref. [4], the factor in front of the term involving $g_{1}$ and $g_{2}$ reads $\beta_{W}^{2} /\left(\beta_{W} \beta_{h}\right)$; it should be $\left(1+\beta_{W}^{2}\right) /\left(\beta_{W} \beta_{h}\right)$. With $\beta_{W}=1$, the prefactors in their Eq. (16) reduce to ours.
} 


$$
\hat{\mu}_{Z}=m_{Z}^{2} / \hat{s}, \quad \hat{\mu}_{W}=m_{W}^{2} / \hat{s}, \quad \hat{\mu}_{h}=m_{h}^{2} / \hat{s}, \quad \hat{\mu}_{H}=m_{H}^{2} / \hat{s},
$$

and the Higgs velocity is $\beta_{h}=\left(1-\hat{\mu}_{h}\right)^{1 / 2}$.

Our approach differs from that of DHZ in that we do not project out the longitudinal degrees of freedom of the intermediate $W$ bosons. Instead, we follow the approach of Ref. [23], where transverse momenta are ignored everywhere except in the $W$ propagators, the integrations over which are approximated as [here $p_{1}$ and $p_{1}^{\prime}$ denote electron and neutrino momenta, respectively, in the process (7.7)]:

$$
\int \mathrm{d}^{2} \mathbf{p}_{1 \perp} \frac{1}{\left[\left(p_{1}-p_{1}^{\prime}\right)^{2}-m_{W}^{2}\right]^{2}} \simeq \frac{\pi\left(1-x_{1}\right)}{m_{W}^{2}},
$$

where $x_{1}$ (and $x_{2}$ ) represents the energy of the $W$. The energy squared of the subprocess is given as $\hat{s}=\left(p_{1} x_{1}\right.$ $\left.+p_{2} x_{2}\right)^{2}=x_{1} x_{2} s=x s$.

The contributions of diagrams (b) $+(\mathrm{c})$, (a) and (d) are given by the terms $g_{0}, g_{3}$ and $g_{4}$, respectively, with $g_{0}(x)$ $=1$, and

$$
\begin{aligned}
g_{3}(x)= & 8 \beta_{h}\left[2 \hat{\mu}_{W}+\left(\hat{\mu}_{h}-\hat{\mu}_{W}\right)^{2}\right] \\
& \times\left[2 \hat{\mu}_{W}+1-3\left(\hat{\mu}_{h}-\hat{\mu}_{W}\right)^{2}\right] \frac{l_{W}}{a_{W}} \\
& +16\left[2 \hat{\mu}_{W}+\left(\hat{\mu}_{h}-\hat{\mu}_{W}\right)^{2}\right]^{2} y_{W}+16 \beta_{h}^{2}\left(1+a_{W}\right)^{2}, \\
g_{4}(x)= & 8 \beta_{h}\left(\hat{\mu}_{h}-\hat{\mu}_{C}\right)^{2}\left[1-3\left(\hat{\mu}_{h}-\hat{\mu}_{C}\right)^{2}\right] \frac{l_{C}}{a_{C}} \\
& +16\left(\hat{\mu}_{h}-\hat{\mu}_{C}\right)^{4} y_{C}+16 \beta_{h}^{2}\left(1+a_{C}\right)^{2},
\end{aligned}
$$

where

$$
\begin{aligned}
& l_{W}=\log \frac{1-2 \hat{\mu}_{h}+2 \hat{\mu}_{W}-\beta_{h}}{1-2 \hat{\mu}_{h}+2 \hat{\mu}_{W}+\beta_{h}}, \\
& y_{W}=\frac{2 \beta_{h}^{2}}{\left(1-2 \hat{\mu}_{h}+2 \hat{\mu}_{W}\right)^{2}-\beta_{h}^{2}}, \\
& a_{W}=-\frac{1}{2}+\hat{\mu}_{h}-\hat{\mu}_{W},
\end{aligned}
$$

and similarly $l_{C}, y_{C}$ and $a_{C}$, with $\hat{\mu}_{W}$ replaced by $\hat{\mu}_{C}$, the latter being defined in terms of the charged Higgs boson mass $m_{H^{+}}$.

The interference between diagrams $(b)+(c)$ and (a) is given by the term $g_{1}$, whereas the interferences between diagrams (b) $+(\mathrm{c})$ and (d), and between (a) and (d) are given by $g_{2}$ and $g_{5}$, respectively. For these interference terms, we find

$$
\begin{aligned}
& g_{1}(x)=8\left[2 \hat{\mu}_{W}+\left(\hat{\mu}_{h}-\hat{\mu}_{W}\right)^{2}\right] l_{W}-4 \beta_{h}\left(1+2 \hat{\mu}_{h}-2 \hat{\mu}_{W}\right), \\
& g_{2}(x)=8\left(\hat{\mu}_{h}-\hat{\mu}_{C}\right)^{2} l_{C}-4 \beta_{h}\left(1+2 \hat{\mu}_{h}-2 \hat{\mu}_{C}\right),
\end{aligned}
$$$$
g_{5}(x)=\frac{\beta_{h}}{4}\left(Z_{W} l_{W}+Z_{C} l_{C}\right)+8 \beta_{h}^{2}\left(1+a_{W}\right)\left(1+a_{C}\right),
$$

with

$$
\begin{aligned}
Z_{W}= & \frac{\left(1+2 a_{W}\right)^{2}}{a_{C}-a_{W}}\left[8 \hat{\mu}_{W}+\left(1+2 a_{W}\right)^{2}\right] \\
& +\frac{\left(1-2 a_{W}\right)^{2}}{a_{C}+a_{W}}\left[8 \hat{\mu}_{W}+\left(1+2 a_{W}\right)^{2}\right], \\
Z_{C}= & -\frac{\left(1+2 a_{C}\right)^{2}}{a_{C}-a_{W}}\left[8 \hat{\mu}_{W}+\left(1+2 a_{C}\right)^{2}\right] \\
& +\frac{\left(1+2 a_{C}\right)^{2}}{a_{C}+a_{W}}\left[8 \hat{\mu}_{W}+\left(1-2 a_{C}\right)^{2}\right] .
\end{aligned}
$$

Our functions $g_{1}-g_{5}$ correspond to those of DHZ, cf. our Eq. (7.10) and their Eq. (16) in [4]. At small $m_{h}$, the cross section is sensitive to small $x$, where the "effective $W W$ approximation" is not well defined, and our results differ from those of DHZ. However, apart from the contributions from small $x$, our results agree with those of DHZ to a precision of $1-5 \%$.

We show in Fig. 15 the $W W$ fusion cross section, at $\sqrt{s}$ $=1.5 \mathrm{TeV}$, as given by Eqs. (7.1) and (7.8), in the limit of no squark mixing, as well as with mixing (as indicated), and with $\tilde{m}=1 \mathrm{TeV}$. The structure is very reminiscent of that of Fig. 14, and for the same reasons. However, the scale is different.

\section{SENSITIVITY TO $\lambda_{H h h}$ AND $\lambda_{h h h}$}

Following [4], we have indicated in the $m_{A}-\tan \beta$ plane the regions where $\lambda_{H h h}$ and $\lambda_{h h h}$ might be measurable, according to criteria analogous to those given there. In Fig. 16, we consider $\sqrt{s}=500 \mathrm{GeV}$, and identify regions according to the following criteria:

(i) Regions where $\lambda_{H h h}$ might become measurable are identified as those where $\sigma(H) \times \mathrm{BR}(H \rightarrow h h)>0.1 \mathrm{fb}$ 

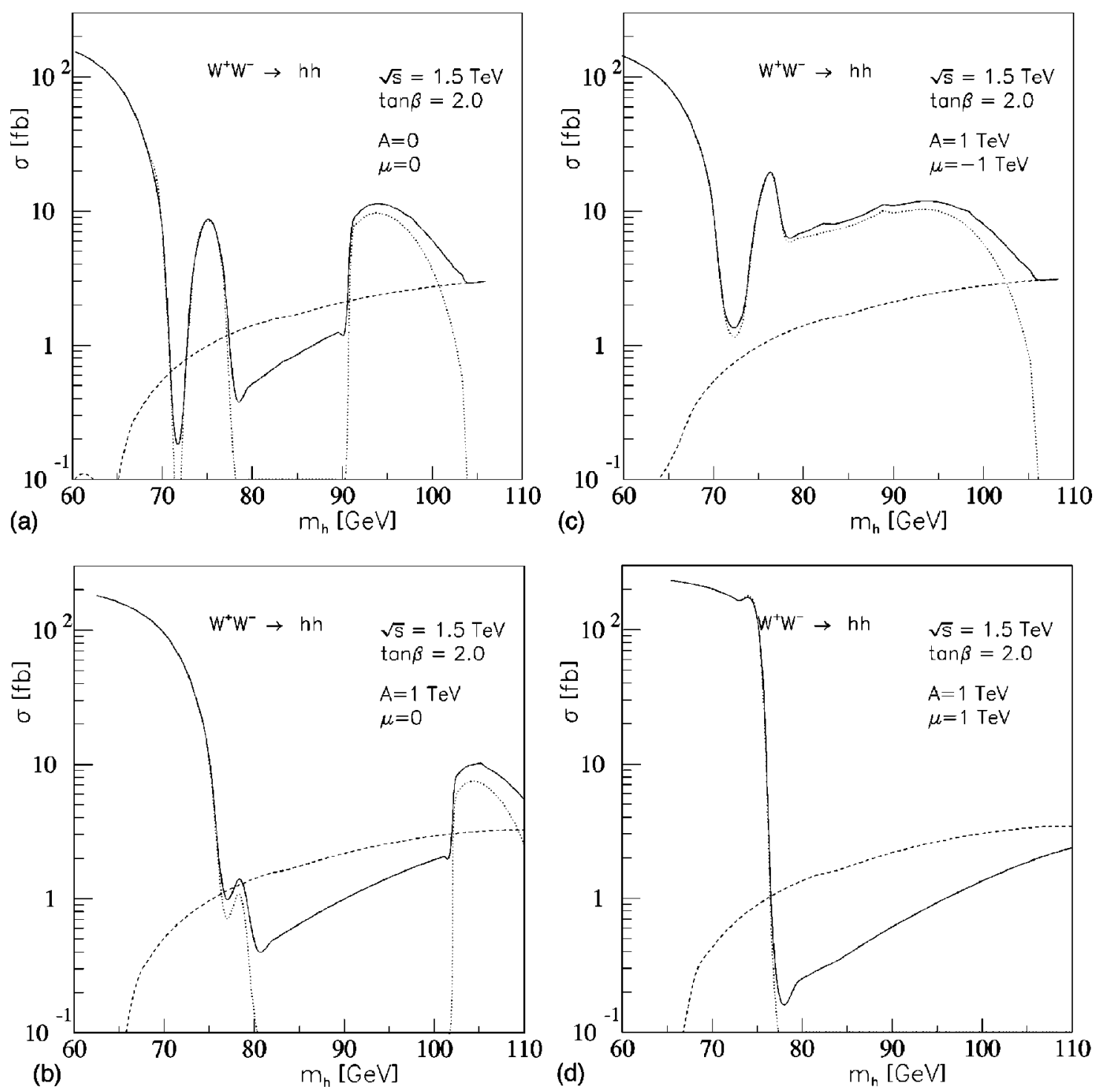

FIG. 15. Cross section $\sigma\left(e^{+} e^{-} \rightarrow \nu_{e} \bar{\nu}_{e} h h\right)$ (via $W W$ fusion) as a function of $m_{h}$, for four cases: (a) no mixing, (b) $-(\mathrm{d}) A=1 \mathrm{TeV}$, $\mu=0,-1$ and $1 \mathrm{TeV}$, as indicated. The dotted curve is the resonant production. The dashed curve gives the decoupling limit.

(solid), with the simultaneous requirement of $0.1<\mathrm{BR}(H$ $\rightarrow h h)<0.9$ (see Figs. 11-13). In view of the recent, more optimistic, view on the luminosity that might become available, we also give the corresponding contours for $0.05 \mathrm{fb}$ (dashed) and $0.01 \mathrm{fb}$ (dotted). For $\sigma(H)$ we take the sum of Eqs. (5.1), (5.2) and (7.1).

(ii) Regions where $\lambda_{h h h}$ might become measurable are those where the continuum $W W \rightarrow h h$ cross section [Eq. (7.8)] is larger than $0.1 \mathrm{fb}$ (solid). Also included are contours at 0.05 (dashed) and $0.01 \mathrm{fb}$ (dotted).

Such regions are given for four cases of the mixing parameters $A$ and $\mu$, as indicated. We have excluded from the plots the region where $m_{h}<72.2 \mathrm{GeV}$, according to the LEP lower bound [11]. This corresponds to low values of $m_{A}$.

We note that with an integrated luminosity of $500 \mathrm{fb}^{-1}$, the contours at $0.1 \mathrm{fb}$ correspond to 50 events per year. This will of course be reduced by efficiencies, but should indicate the order of magnitude that can be reached.
At $\sqrt{s}=500 \mathrm{GeV}$, with a luminosity of $500 \mathrm{fb}^{-1}$ per year, the trilinear coupling $\lambda_{H h h}$ is accessible in a considerable part of the $m_{A}-\tan \beta$ parameter space: at $m_{A}$ of the order of $200-300 \mathrm{GeV}$ and $\tan \beta$ up to the order of 5. With increasing luminosity, the region extends somewhat to higher values of $m_{A}$.

At values of $m_{A}$ below $100 \mathrm{GeV}$, there is also a narrow band where $\lambda_{H h h}$ is accessible.

The "steep"' edge around $m_{A} \simeq 200 \mathrm{GeV}$ (where increased luminosity does not help) is determined by the vanishing of $\mathrm{BR}(H \rightarrow h h)$, see Fig. 13 .

The coupling $\lambda_{h h h}$ is accessible in a much larger part of this parameter space, but with a moderate luminosity, "large" values of $\tan \beta$ are accessible only if $A$ is small.

In Fig. 17, we consider $\sqrt{s}=1.5 \mathrm{TeV}$, and present the analogous contours. Here, for the case of $\lambda_{H h h}$ we demand $\sigma(H) \times \mathrm{BR}(H \rightarrow h h)>0.5 \mathrm{fb}$ (solid) and $0.1 \mathrm{fb}$ (dashed), and 

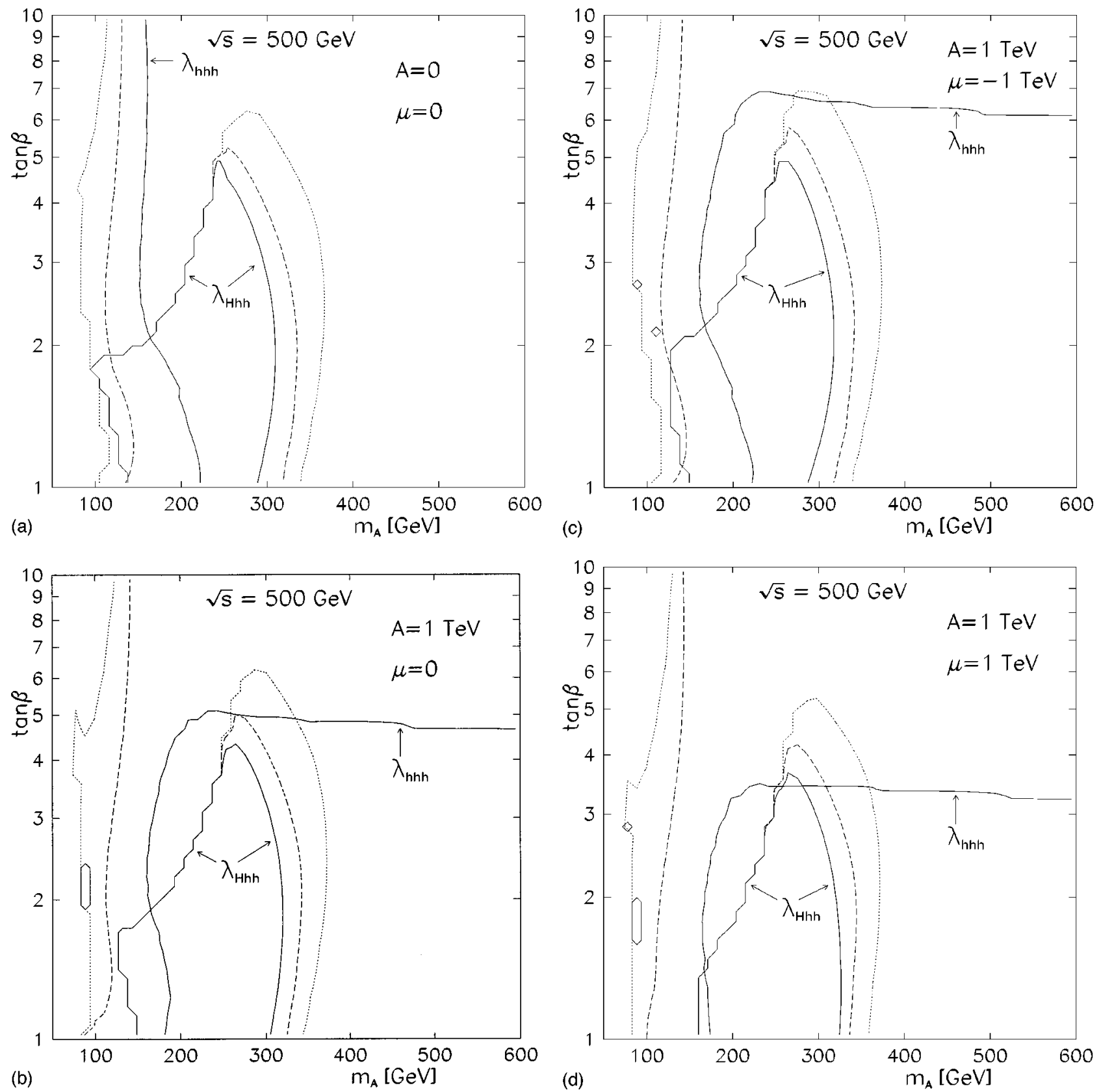

FIG. 16. Regions where trilinear couplings $\lambda_{H h h}$ and $\lambda_{h h h}$ might be measurable at $\sqrt{s}=500 \mathrm{GeV}$. Inside contours labelled $\lambda_{H h h}$, $\sigma(H) \times \mathrm{BR}(H \rightarrow h h)>0.1 \mathrm{fb}$ (solid), while $0.1<\mathrm{BR}(H \rightarrow h h)<0.9$. Inside (to the right or below) contour labelled $\lambda_{h h h}$, the continuum $W W \rightarrow h h$ cross section exceeds $0.1 \mathrm{fb}$ (solid). Analogous contours are given for 0.05 (dashed) and $0.01 \mathrm{fb}$ (dotted). Four cases of mixing are considered, with $A=0$ or $1 \mathrm{TeV}$, and $\mu=0$ or $\pm 1 \mathrm{TeV}$, as indicated.

for the case of $\lambda_{h h h}$ we require the corresponding cross section [Eq. (7.8)] to be larger than $0.5 \mathrm{fb}$ (solid) and $0.1 \mathrm{fb}$ (dashed). If a luminosity corresponding to these cross sections becomes available at $\sqrt{s}=1.5 \mathrm{TeV}$, a somewhat larger region than at $\sqrt{s}=500 \mathrm{GeV}$ is accessible in the $m_{A}-\tan \beta$ plane.

It should be stressed that the requirements discussed here are necessary, but not sufficient conditions for the trilinear couplings to be measurable. We also note that there might be sizable corrections to the $W W$ approximation, and that it would be desirable to incorporate the dominant two-loop corrections to the trilinear couplings in the calculations.

\section{CONCLUSIONS}

We have carried out a detailed investigation of the possibility of measuring the MSSM trilinear couplings $\lambda_{H h h}$ and $\lambda_{h h h}$ at an $e^{+} e^{-}$collider. Where there is an overlap, we have confirmed the results of Ref. [4]. Our emphasis has been on taking into account all the parameters of the MSSM Higgs 

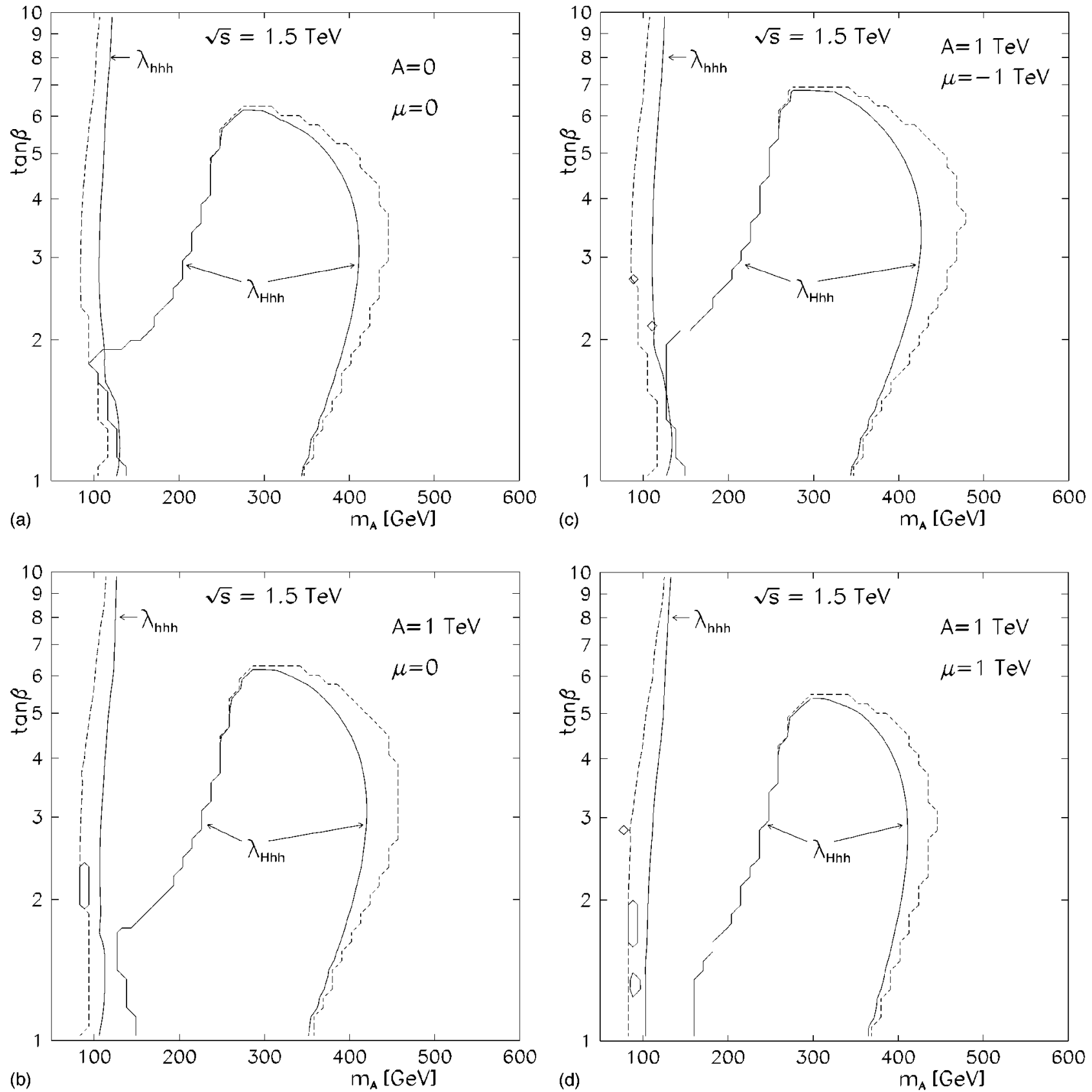

FIG. 17. Regions where trilinear couplings $\lambda_{H h h}$ and $\lambda_{h h h}$ might be measurable at $\sqrt{s}=1.5 \mathrm{TeV}$. The contours correspond to $0.5 \mathrm{fb}$ (solid) and $0.1 \mathrm{fb}$ (dashed). All other parameters are the same as in Fig. 16.

sector. We have studied the importance of mixing in the squark sector, as induced by the trilinear coupling $A$ and the bilinear coupling $\mu$.

At moderate energies $(\sqrt{s}=500 \mathrm{GeV})$ the range in the $m_{A}-\tan \beta$ plane that is accessible for studying $\lambda_{H h h}$ changes quantitatively for non-zero values of the parameters $A$ and $\mu$. As far as the coupling $\lambda_{h h h}$ is concerned, however, there is a qualitative change from the case of no mixing in the squark sector. If $A$ is large, then high luminosity is required to reach "high" values of $\tan \beta$. At higher energies $(\sqrt{s}=1.5 \mathrm{TeV})$, the mixing parameters $A$ and $\mu$ change the accessible region of the parameter space only in a quantitative manner.

\section{ACKNOWLEDGMENTS}

P.O. would like to thank the DESY Theory Group and the CERN Theory Division, whereas P.N.P. would like to thank the University of Bergen, for kind hospitality while parts of this work were finished. It is also a pleasure to thank Abdel Djouadi, Wolfgang Hollik, Bernd Kniehl, Conrad Newton and Peter Zerwas for valuable discussions and advice. This research was supported by the Research Council of Norway, and (P.N.P.) by the University Grants Commission, India under project number 10-26/98(SR-I). 
[1] J. F. Gunion, H. E. Haber, G. Kane, and S. Dawson, The Higgs Hunter's Guide (Addison-Wesley, New York, 1990).

[2] For reviews, see H.-P. Nilles, Phys. Rep. 110, 1 (1984); H. E. Haber and G. L. Kane, ibid. 117, 75 (1985); R. Barbieri, Riv. Nuovo Cimento 11, 1 (1988).

[3] T. Plehn, M. Spira, and P. M. Zerwas, Nucl. Phys. B479, 46 (1996).

[4] A. Djouadi, H. E. Haber, and P. M. Zerwas, Phys. Lett. B 375, 203 (1996); and erratum (to be published).

[5] See, e.g., ECFA/DESY LC Physics Working Group, E. Accomando et al., Phys. Rep. 299, 1 (1998). The luminosity quoted there is now believed to be too conservative.

[6] J. Ellis, G. Ridolfi, and F. Zwirner, Phys. Lett. B 257, 83 (1991); Y. Okada, M. Yamaguchi, and T. Yanagida, Prog. Theor. Phys. 85, 1 (1991); H. E. Haber and R. Hempfling, Phys. Rev. Lett. 66, 1815 (1991).

[7] J. Ellis, G. Ridolfi, and F. Zwirner, Phys. Lett. B 262, 477 (1991).

[8] R. Hempfling and A. H. Hoang, Phys. Lett. B 331, 99 (1994); M. Carena, J. R. Espinosa, M. Quirós, and C. E. M. Wagner, ibid. 355, 209 (1995); M. Carena, M. Quirós, and C. E. M. Wagner, Nucl. Phys. B461, 407 (1996); S. Heinemeyer, W. Hollik, and G. Weiglein, Phys. Rev. D 58, 091701 (1998).

[9] B. Kileng, P. Osland, and P. N. Pandita, Z. Phys. C 71, 87 (1996); B. Kileng, P. Osland, and P. N. Pandita, in Xth International Workshop: High Energy Physics and Quantum Field Theory, Proceedings of the Conference, Zvenigorod, Russia, 1995, edited by B. B. Levtchenko and V. I. Savrin (Moscow University Press, Moscow, 1996), p. 167, hep-ph/9601284; B. Kileng, P. Osland, and P. N. Pandita, in Proceedings of the International Workshop on Quantum Systems: New Trends and Methods, Minsk, Belarus, 1996, edited by Y. S. Kim, L. M. Tomil'chik, I. D. Feranchuk, and A. Z. Gazizov (World Scientific, Singapore, 1997), p. 231, hep-ph/9608315.

[10] ALEPH Collaboration, R. Barate et al., Phys. Lett. B 412, 173 (1997).
[11] ALEPH Collaboration, R. Barate et al., Phys. Lett. B 440, 419 (1998).

[12] M. Carena, P. H. Chankowski, S. Pokorski, and C. E. M. Wagner, Phys. Lett. B 441, 205 (1996).

[13] V. Barger, M. S. Berger, A. L. Stange, and R. J. N. Phillips, Phys. Rev. D 45, 4128 (1992).

[14] A. Sirlin and R. Zucchini, Nucl. Phys. B266, 389 (1986).

[15] A. Djouadi, J. Kalinowski, and P. M. Zerwas, Z. Phys. C 70, 435 (1996).

[16] G. Pócsik and G. Zsigmond, Z. Phys. C 10, 367 (1981).

[17] J. F. Gunion, L. Roszkowski, A. Turski, H. E. Haber, G. Gamberini, B. Kayser, S. F. Novaes, F. Olness, and J. Wudka, Phys. Rev. D 38, 3444 (1988).

[18] H. E. Haber, in Proceedings of the Conference on Perspectives for Electroweak Interactions in $e^{+} e^{-}$Collisions, Ringberg (Tegernsee), Germany, 1995, edited by B. A. Kniehl (World Scientific, Singapore, 1995), p. 219.

[19] For recent references, see A. Djouadi, P. Janot, J. Kalinowski, and P. M. Zerwas, Phys. Lett. B 376, 220 (1996); A. Bartl, H. Eberl, K. Hidaka, T. Kon, W. Majerotto, and Y. Yamada, in Beyond the Standard Model, 5th International Conference on Physics Beyond the Standard Model, Balholm, Norway, edited by G. Eigen, P. Osland, and B. Stugu (AIP, Woodbury, NY, 1997), p. 494, hep-ph/9709253.

[20] A. Djouadi, J. Kalinowski, and P. M. Zerwas, in Proceedings, Workshop on $e^{+} e^{-}$Collisions at $500 \mathrm{GeV}$ : The Physics Potential, Munich-Annecy-Hamburg (DESY Report No. 92123A, Hamburg, 1992).

[21] Djouadi, Haidt, Kniehl, Mele, and Zerwas (Ref. [20]).

[22] R. N. Cahn and S. Dawson, Phys. Lett. 136B, 196 (1984); S. Dawson, Nucl. Phys. B249, 42 (1984); M. Chanowitz and M. K. Gaillard, Phys. Lett. 142B, 85 (1984); I. Kuss and H. Spiesberger, Phys. Rev. D 53, 6078 (1996).

[23] G. Altarelli, B. Mele, and F. Pitolli, Nucl. Phys. B287, 205 (1987). 